\title{
Long-Term Epigenetic Alterations in a Rat Model of Gulf War Illness
}

Lisa M. Pierce ${ }^{\mathrm{a}}$, Wendy E. Kurata ${ }^{\mathrm{a}}$, Karen W. Matsumoto ${ }^{\mathrm{a}}$, Margaret E. Clark ${ }^{\mathrm{b}}$, Douglas M. Farmer $^{\mathrm{b}}$

${ }^{a}$ Department of Clinical Investigation, Tripler Army Medical Center, 1 Jarrett White Road, Honolulu, Hawaii, 96859 USA

${ }^{\mathrm{b}}$ Department of General Surgery, Tripler Army Medical Center, 1 Jarrett White Road, Honolulu, Hawaii 96859 USA

The views expressed in this manuscript are those of the authors and do not reflect the official policy or position of the Department of the Army, Department of Defense, or the US Government.

\section{Corresponding Author Contact Information:}

Name: $\quad$ Lisa M. Pierce, DSc

Address: $\quad$ Tripler Army Medical Center Department of Clinical Investigation 1 Jarrett White Road Honolulu, Hawaii 96859-5000

Phone: $\quad$ (808) 433-5219

Fax: $\quad$ (808) 422-7160

Email: $\quad$ lisa.m.pierce.civ@ mail.mil 


\begin{abstract}
Gulf War Illness (GWI) is a chronic, multisymptom illness that affects $25 \%$ of the 700,000 US veterans deployed to the Persian Gulf during the 1990-1991 Gulf War. Central nervous system impairments are among the most common symptoms reported, including memory dysfunction and depression. After 25 years, the diagnosis remains elusive, useful treatments are lacking, and the cause is poorly understood, although exposures to pyridostigmine bromide (PB) and pesticides are consistently identified to be among the strongest risk factors. Epigenetic changes including altered microRNA (miRNA) expression and DNA methylation play an important role in in learning, memory, and emotion regulation and have been implicated in various neurological disorders. In this study, we used an established rat model of GWI to determine whether 1) chronic alterations in miRNA expression and global DNA methylation and DNA hydroxymethylation are mechanisms involved in the pathobiology of GWI, and 2) plasma exosome small RNAs may serve as potential noninvasive biomarkers of this debilitating disease. One year after a 28-day exposure regimen of PB, DEET ( $N, N$-diethyl-3-methylbenzamide), permethrin, and mild stress, expression of 84 mature miRNAs and global 5-methylcytosine $(5 \mathrm{mC})$ and 5-hydroxymethylcytosine $(5 \mathrm{hmC})$ content were analyzed in the brains of GWI rats and vehicle controls by PCR array and enzyme-linked immunosorbent assay, respectively. Plasma exosome RNA next-generation sequencing analysis was performed in pooled samples to discover potential noninvasive biomarkers. We found that combined exposure to low doses of GW-related chemicals and mild stress caused epigenetic modifications in the brain that persisted one year after exposure, including increased expression of miR-124-3p and miR-29b-3p in the hippocampus and regional alterations in global $5 \mathrm{mC}$ and $5 \mathrm{hmC}$ content. $\mathrm{GW}$-relevant exposures also induced the differential expression of two piwi-interacting RNAs (piRNAs) in circulation (piR-007899 and piR-019162). Results from this study implicate a role for epigenetic alterations in GWI. Evaluation of the diagnostic potential of plasma exosome RNAs in veterans with GWI is warranted.
\end{abstract}

Keywords: Gulf War Illness, Epigenetics, microRNA, DNA methylation, Pyridostigmine bromide, Pesticides 


\section{Introduction}

Gulf War Illness (GWI) is a chronic, multisymptom illness that affects approximately 25$30 \%$ of the 700,000 U.S. veterans deployed to the Persian Gulf during the 1990-1991 Gulf War [Institute of Medicine, 1996; Research Advisory Committee on Gulf War Veterans' Illnesses (RAC), 2008, 2014]. Neurological health effects including concentration problems, learning and memory difficulties, and depression are among the most prominent symptoms reported; additional complaints include severe fatigue, headaches, musculoskeletal pain, gastrointestinal issues, skin rashes, poor coordination, sleep disturbances, and weakened immune function (Kang et al., 2009; Li B et al., 2011; Odegard et al., 2013; Smith et al., 2013). Biological evidence for central nervous system involvement has been provided by behavioral and functional imaging studies in ill GW veterans which revealed chronically altered structure and function of the hippocampus, a brain region critical for learning and memory formation (Menon et al., 2004; Vythilingham et al., 2005; Li X et al., 2011; Rayhan et al, 2013; Hubbard et al., 2014). Reduced white and gray matter volumes in cortical areas have also been reported in structural MRI studies (Chao et al., 2011; Rahan et al., 2013). Although some progress has been made, useful diagnostic indicators and treatments for GWI are currently lacking after 25 years, and underlying cellular and molecular mechanisms involved in its pathobiology remain poorly understood (White et al., 2016).

While several etiologies have been proposed for GWI, epidemiological studies have consistently identified exposure to cholinesterase-inhibiting chemicals during deployment to be among the strongest risk factors for the persistent symptoms affecting GW veterans (RAC, 2008, 2014; Steele et al., 2012; Kerr, 2015; White et al., 2016). These include the ingestion of pyridostigmine bromide (PB) pills issued as a prophylactic treatment against nerve agent attack and the overuse of insect repellants and pesticides such as DEET ( $N, N$-diethyl-3methylbenzamide) and permethrin. PB is a reversible acetylcholinesterase inhibitor which does not normally cross the blood-brain barrier, but may enter the brain after exposure to stress or pesticides (Friedman et al., 1996; Abdel-Rahman et al., 2002). DEET also inhibits cholinesterase activity, and while generally considered to possess low toxicity at physiological levels, excessive exposure has resulted in tremors, restlessness, difficulty in speech, seizures, impairment of cognitive function, and coma (Corbel et al., 2009). Permethrin toxicity is due to the sustained 
opening of sodium channels, with symptoms including tremors, hyperactivity, ataxia, convulsions, and paralysis (Meyer et al., 2008). In addition, detonations at Khamisiyah and other Iraqi weapons facilities may have contributed low-level exposure to cholinesterase-inhibiting organophosphate nerve agents such as sarin (RAC, 2008, 2014). It is thought that the accumulation of acetylcholine at cholinergic synapses leads to long-term cholinergic suppression and may cause the central and peripheral nervous system dysfunction observed in GWI (Hubbard et al., 2014; Zakirova et al., 2015).

Epidemiological findings suggesting a role for neurotoxic chemicals as causative agents are supported experimentally by neuropathological and neurobehavioral changes observed in animal models of GWI (Abdel-Rahman et al., 2002; Abdel-Rahman et al., 2004; Abdullah et al., 2011, 2012; Parihar et al., 2013; Hattiangady et al., 2014; Ojo et al., 2014; O'Callaghan et al., 2015; Zakirova et al., 2015). Exposure of rats to physiologically relevant levels of PB, DEET, permethrin, and mild stress resulted in memory dysfunction and depression, reduced hippocampal neurogenesis, and hippocampal neuron loss (Parihar et al., 2013; Hattiangady et al., 2014; Megahed et al., 2015). Exposure of mice to PB and permethrin similarly caused long-term cognitive deficits, an increase in brain acetylcholine levels, and presynaptic vesicle loss in the hippocampus and cerebral cortex, indicating impairment of synaptic integrity and perturbation of the cholinergic system (Zakirova et al., 2015). Decreased hippocampal neurogenesis and improper synapse formation have been linked to cognitive impairment, neuropsychiatric and neurodegenerative disorders, and depression (Snyder et al., 2001; Sun et al., 2011; GarzaManero et al., 2014).

Although rodent models of GWI have revealed delayed and persistent effects on cognition, synaptic transmission, axonal transport, and alterations in genomic, proteomic, lipidomic, and metabolomic profiles, the effects of epigenetic modifications in the pathobiology of GWI have yet to be explored (Kerr, 2015; White et al., 2016). A major US report updated in 2014 recommended that research using animal models of GWI should "continue to examine the immediate, delayed, and persistent effects of acute exposures to chemicals and chemical mixtures" and advocated "epigenetic and genetic approaches to research" (RAC, 2014). Epigenetic changes modify the expression of genes but not the sequence of DNA, and serve as an important interface between genes and the environment. Environmental exposures have consequences for gene expression through mechanisms including DNA methylation, histone 
modifications, and noncoding RNAs (Reamon-Buettner et al., 2008). Emerging evidence indicates that epigenetic alterations may mediate toxicity from pesticides and may be involved in the development of depression (Manikkam et al., 2012; Collotta et al., 2013; Vialou et al., 2013; Januar et al., 2015). Stress has also been shown to induce epigenetic changes in the brain, resulting in reduced expression of genes involved in neural plasticity (Stankiewicz et al., 2013).

The significance of epigenetic mechanisms in the normal functioning of the central nervous system and the onset of memory impairments and other neurological disorders is becoming increasingly apparent (Mikaelsson and Miller, 2011; Ruzicka, 2015). Two of the most wellstudied epigenetic mechanisms known to modulate synaptic plasticity, neurotransmission, and neurogenesis, processes essential for learning and memory formation and emotion regulation, include microRNA (miRNA) expression and methylation of DNA cytosines at the 5-position (Miller and Sweatt, 2007; Guo et al., 2011; Sun et al., 2011; Salta and De Strooper, 2012). miRNAs are small (about 22 nucleotides in length), single-stranded, non-protein-coding RNAs that control gene expression through post-transcriptional mechanisms (Tal and Tanguay, 2012). Although methylation of genomic DNA was once considered to be a relatively stable epigenetic marker in post-mitotic cells, 5-methylcytosine $(5 \mathrm{mC})$ and 5-hydroxymethylcytosine $(5 \mathrm{hmC}$, a stable intermediate between methylation and demethylation) are now known to be dynamic and reversible post-translational modifications in the adult nervous system that are highly enriched in genes involved in synaptic regulation (Miller and Sweatt, 2007; Guo et al., 2011; Chen et al., 2012; Wen and Tang, 2014).

In this study we used an established rat model of GWI to determine whether exposure to a combination of GW-relevant neurotoxic chemicals and stress induces chronic alterations in miRNA expression and global DNA methylation and hydroxymethylation status in the brain. Epigenetic changes were evaluated in rats one year after exposure to mimic the persistent effects suffered by current veterans with GWI who are presently 25 years post-exposure. Initial studies were also conducted to examine the utility of circulating miRNAs and plasma exosome RNAs to serve as potential noninvasive biomarkers of GWI. Exosomes are small $(30-120 \mathrm{~nm})$ extracellular vesicles of endocytic origin released from cells that contain a cargo of proteins and nucleic acids (Vlassov et al., 2012; Kalani et al., 2014; Kawikova and Askenase, 2015). Nextgeneration sequencing technologies have identified various small noncoding RNAs besides 
miRNAs in exosomes, many of which are involved in cell-cell communication and may represent novel cell signaling factors in the blood (Huang et al., 2013; Revenfeld et al., 2014).

\section{Materials and methods}

\subsection{Chemicals}

PB ( $\geq 98 \%$ ), permethrin (analytical standard containing a $98.3 \%$ mixture of $26.7 \%$ cis and $71.6 \%$ trans isomers), and DEET ( $\geq 97 \%$ ) were purchased from Sigma-Aldrich (St. Louis, MO). All other reagents were of the highest purity available from commercial vendors.

\subsection{Animals}

Young adult male Sprague-Dawley rats (9 weeks of age, approximately $300 \mathrm{~g}$ ) underwent the 28-day GW exposure protocol described below and were euthanized one year after the completion of the exposure regimen. Rats were obtained from Taconic (Germantown, NY) and were housed in polycarbonate cages in HEPA-filtered laminar airflow racks with wood chip bedding under controlled lighting conditions ( $12 \mathrm{~h}$ light/12 $\mathrm{h}$ dark). The rats were allowed free access to standard laboratory diet and tap water. The study protocol was approved by the Institutional Animal Care and Use Committee at Tripler Army Medical Center and investigators complied with the policies as prescribed in the USDA Animal Welfare Act and the National Research Council's Guide for the Care and Use of Laboratory Animals. Facilities are fully accredited by the Association for Assessment and Accreditation of Laboratory Animal Care International.

\subsection{GW exposure protocol}

The exposure regimen of Abdel-Rahman et al. involving physiologically-relevant doses of dermal DEET, dermal permethrin, and oral PB followed by 5 minutes of restraint stress was utilized (Abdel-Rahman et al., 2002; Abdel-Rahman et al., 2004; Parihar et al., 2013; Hattiangady et al., 2014). GWI rats ( $\mathrm{n}=33)$ were treated daily with PB $(1.3 \mathrm{mg} / \mathrm{kg} / \mathrm{day}$, oral in water), DEET (40 mg/kg/day, dermal in $70 \%$ ethanol), and permethrin $(0.13 \mathrm{mg} / \mathrm{kg} / \mathrm{day}$, dermal in $70 \%$ ethanol) for 28 days. This exposure regimen has been designed to simulate the daily exposure experienced by veterans during the Gulf War (Abdel-Rahman et al., 2002; Abdel- 
Rahman et al., 2004). GWI rats were also subjected to 5 minutes of restraint stress every day for the duration of the exposure period. Restraint occurred after the chemical treatment by placing the rats in a Plexiglas cylinder. Vehicle-treated control rats $(n=32)$ were treated with a dermal application of $70 \%$ ethanol and oral water daily for 28 days using an equal volume of vehicle as that given to GWI rats. Oral doses were given by gavage ( $1 \mathrm{ml} / \mathrm{kg}$ body weight), while dermal

applications (about $0.1 \mathrm{ml}$ ) were applied to the back of the neck on a $2.5 \mathrm{~cm}^{2}$ area preshaved with clippers. Body weight measurements were obtained weekly throughout the exposure regimen and every two weeks during the year-long post-treatment survival period.

\subsection{Tissue and blood collection}

One year after completion of the exposure period, rats designated for miRNA, mRNA, DNA methylation, and cytokine protein analyses were euthanized by lethal injection of a pentobarbital-based euthanasia solution, and brains were removed rapidly. Brains were dissected in half sagittally using an ice cold rat brain slicer (Zivic Instruments, Pittsburgh, PA), and the hippocampus, overlying cerebral cortex, and cerebellum were separated. Regional dissection was aided with the use of a rat brain stereotaxic atlas and published diagrams (Heffner et al., 1980; Paxinos and Watson, 1998). For animals designated for miRNA analysis, left and right brain tissues were stored in RNALater solution (Life Technologies Corp., Grand Island, NY) at $-80^{\circ} \mathrm{C}$ until RNA isolation was performed. For animals designated for global DNA methylation and hydroxymethylation detection, mRNA analysis, and cytokine protein measurement, left brain tissues were snap frozen in liquid nitrogen for protein analysis and right brain tissues were stored in RNALater solution at $-80^{\circ} \mathrm{C}$ until total RNA and genomic DNA extraction.

Rats designated for histopathological analysis were deeply anesthetized with sodium pentobarbital $(100 \mathrm{mg} / \mathrm{kg})$ and perfused through the heart with saline followed by $4 \%$ paraformaldehyde (approximately $400 \mathrm{ml}$ each solution). Brains were removed, post-fixed 72 hours in $4 \%$ paraformaldehyde, cut into approximately $4 \mathrm{~mm}$ coronal blocks using a rat brain slicer, and embedded in paraffin.

To determine whether putative identified differentially expressed brain miRNAs were detectable in circulation and whether they and/or plasma exosome RNAs could potentially serve as novel noninvasive biomarkers of GWI, blood was collected from the same rats described 
above immediately prior to euthanasia and brain harvest. Blood was drawn from the heart in deeply anesthetized rats either by cardiac puncture or via the right atrium during perfusionfixation. Blood was collected into ethylenediaminetetraacetic acid tubes and spun 10 minutes at $1900 \times \mathrm{g}(3000 \mathrm{rpm})$ at $4^{\circ} \mathrm{C}$. Plasma was transferred to new RNAse-free tubes, spun 10 minutes at $16000 \times \mathrm{g}$, aliquoted, and stored at $-80^{\circ} \mathrm{C}$ until use.

\section{5. miRNA expression profiling using a real-time PCR array}

Samples of hippocampus, overlying cerebral cortex, and cerebellum were homogenized in QIAzol lysis reagent (Qiagen, Valencia, CA) using an Omni GLH homogenizer (Omni International, Keensaw, GA), and total RNA (including miRNA) was purified using the miRNeasy Kit (Qiagen) according to the manufacturer's instructions. Extracted RNA was quantified using a spectrophotometer and stored at $-80^{\circ} \mathrm{C}$. cDNA was generated from $250 \mathrm{ng}$ RNA using the miScript II RT kit with miScript HiSpec Buffer for mature miRNA detection and analyzed using the miScript Rat Neurological Development and Disease miRNA PCR Array (Qiagen) by means of a Bio-Rad iCycler real-time PCR Detection System (Bio-Rad Laboratories, Hercules, CA) per manufacturer instructions. This miRNA PCR array analyzes 84 different mature miRNAs known to be altered in neurological disorders or involved in neuronal development and includes RNA quality controls and internal housekeeping genes to normalize the data for the amount of RNA added to each reverse transcription reaction. Expression profiles were determined using miRNA purified from a single rat per array, and arrays were repeated with 7-9 different rats in each experimental group for the hippocampus, cortex, and cerebellum. Raw threshold cycle $\left(\mathrm{C}_{\mathrm{T}}\right)$ values were normalized to the small noncoding RNAs SNORD68 and SNORD96A on the array using software from Qiagen (miScript miRNA PCR Array Data Analysis web portal) since they presented similar expression levels across individuals and between groups. The analysis tool was used to perform relative quantification using the $\Delta \Delta \mathrm{C}_{\mathrm{T}}$ method to reveal statistically significant fold differences. Differentially expressed miRNAs were considered significant using a cutoff value $>2.5$ fold change and $\mathrm{p}<0.01$. A complete list of miRNAs contained in the array can be viewed on the following link: http://www.sabiosciences.com/mirna_pcr_product/HTML/MIRN-107Z.html.

To confirm mature miRNA expression differences identified using the miRNA PCR array, quantitative real-time PCR was repeated using Qiagen's miRNA-specific primer assays (for rat 
miR-124-3p, miR-29b-3p, miR-22-3p, miR-33-5p, miR-381-3p, and miR-488-3p) by means of a Bio-Rad CFX96 real-time PCR Detection System according to the manufacturer's instructions. Primer efficiency was tested by serial dilution. cDNA was prepared from $1 \mu \mathrm{g}$ RNA using the miScript II RT Kit and used as a template (diluted 1:200) in real-time PCR with a miScript Primer Assay and the miScript SYBR Green PCR Kit. Assays were performed on miRNA extracted from the hippocampus of 9 GWI and 9 control samples from the contralateral side used in the PCR array. Standard curves for each primer assay were generated in each PCR run using 10-fold dilutions enabling the determination of miRNA copy number using software from BioRad. $\mathrm{C}_{\mathrm{T}}$ values from duplicate measurements of each sample were averaged and the expression of each miRNA was normalized to the levels of SNORD96A (which showed the highest stability in our prior analyses) within each sample. Relative expression levels were determined by dividing the average ratio (copy number miRNA of interest/copy number SNORD96A) of the normalized GWI samples by the average ratio of the normalized control samples to obtain a fold difference in miRNA expression between experimental groups. A fold change greater than 1.5 and $\mathrm{p}<0.05$ was considered to be confirmation of differentially expressed miRNAs identified on the array.

\section{6. miRNA target prediction and pathway analysis}

To determine the biological significance of differentially expressed brain miRNAs, the miRNA target prediction tool miRecords (http://c1.accurascience.com/miRecords/, accessed December 2015) was used to generate a list of mRNA targets for the miRNAs determined to be differentially expressed between GWI and control rats (miR-124-3p and miR-29b-3p; Xiao et al., 2009). The validated targets component of miRecords is a large, high-quality database of experimentally validated miRNA targets which contains 2705 records of interactions between 644 miRNAs and 1901 target genes in 9 animal species. The predicted targets component of miRecords is an integration of predicted miRNA targets produced by 11 established miRNA target prediction programs (DIANA-microT, MicroInspector, miRanda, MirTarget2, miTarget, NBmiRTar, PicTar, PITA, RNA22, RNAhybrid, and TargetScan/TargertScanS). Target prediction was determined separately for both rat and human species because mature miR-124 and miR-29b are highly conserved across mammals, having the identical nucleotide sequence in humans, rats, and mice (Jiao et al., 2010; Pan-Vazquez et al., 2015). Targets were selected based 
on the following criteria: validated or predicted with agreement from $\geq 3 / 11$ target prediction programs used by miRecords when rat miRNAs were searched (no results were obtained using a cutoff of 4 target prediction programs for rat miRNAs), and $\geq 6 / 11$ target prediction programs used by miRecords when a separate analysis using human miRNAs was conducted.

To identify involved pathways, gene enrichment analyses was performed using the Database for Annotation, Visualization and Integrated Discovery (DAVID; https://david.ncifcrf.gov/, accessed December 2015; Huang et al., 2009a, 2009b). Pathway classification within DAVID used the Kyoto Encyclopedia of Genes and Genomes (KEGG) Pathway database (http://www.genome.jp/kegg/pathway.html), PANTHER (http://www.pantherdb.org/pathway/), and REACTOME (http://www.reactome.org/PathwayBrowser/). Enrichment statistics were adjusted for multiple hypothesis testing by the Benjamini correction.

\subsection{Quantification of identified differentially expressed brain miRNAs in circulation}

To evaluate the diagnostic potential of differentially expressed brain miRNAs in circulation, thawed plasma samples were spun 5 minutes at $16000 \times \mathrm{g}$ at $4^{\circ} \mathrm{C}$ to remove cryoprecipitates and were transferred to new RNAse-free tubes. RNA was isolated from $200 \mu$ l plasma using the miRNeasy Serum/Plasma Kit (Qiagen) according to the manufacturer's protocol. A synthetic spike-in control (miRNeasy Serum/Plasma Spike-in Control, a Caenorhabditis elegans miR-39 miRNA mimic) was added before nucleic acid isolation to enable normalization of real-time PCR miRNA expression data and monitor plasma miRNA recovery rates. cDNA was synthesized using the miScript II RT kit using a fixed volume of RNA preparation and was analyzed using miScript Primer Assays (for rat miR-124-3p and miR-29b-3p) according to the manufacturer's instructions. RNA recovery was assessed by comparing $\mathrm{C}_{\mathrm{T}}$ values to a standard curve of the synthetic spike-in control miRNA generated independently from the RNA purification procedure. Raw $\mathrm{C}_{\mathrm{T}}$ values from duplicate or triplicate measurements of each sample were averaged and normalized against the mean of the synthetic spike-in control within each sample to adjust for differences in RNA extraction and reverse transcription efficiencies. Relative quantification using the $\Delta \Delta \mathrm{C}_{\mathrm{T}}$ method was used to determine statistically significant fold differences between individual plasma samples available from $30 \mathrm{GWI}$ and 27 vehicle control animals. 


\subsection{Plasma exosome RNA sequencing (RNA-seq) analysis}

To expand the search for miRNAs and other small noncoding RNAs that could serve as potential novel biomarkers of GWI, we conducted a preliminary analysis to perform nextgeneration sequencing (NGS) of plasma exosome RNAs isolated from GWI rats and vehicle controls. Plasma samples were pooled from available samples (24 control rats and 29 GWI rats) to identify robust differences between groups and to cancel out individual variances between samples. Exosomes were isolated from $500 \mu \mathrm{l}$ plasma using ExoQuick exosome precipitation solution [System Biosciences Inc. (SBI), Mountain View, CA] according to the manufacturer's instructions, and exosome pellets were mailed to SBI for RNA-seq analysis utilizing SBI's Exosome RNA NGS Service. Briefly, exosome RNA was purified and RNA yield was determined using an Agilent Bioanalyzer 2100 System with the Agilent Small RNA Kit (Agilent Technologies, Santa Clara, CA). Illumina NGS libraries were prepared and sequenced using an Illumina HiSeq2000 sequence analyzer (Illumina Inc., San Diego, CA). Data analysis occurred with the use of the Maverix Exosome RNA-seq Analysis platform (Maverix Biomics Inc., San Mateo, CA) using the rat reference RGSC 5.0/rn5 assembly (UCSC Genome Bioinformatics). The web-based analysis service included library sequence quality control metrics, normalization of raw sequence reads, data analysis for relative RNA abundance and identity, and differential expression analysis. Differentially expressed plasma exosome RNAs were reported as $\log 2$ fold change with significance considered at $\mathrm{p}<0.05$ (adjusted for multiple comparisons).

\subsection{Global DNA methylation and hydroxymethylation detection}

Samples of hippocampus, cerebral cortex, and cerebellum from 7 GWI rats and 8 vehicle control rats were homogenized using an Omni GLH homogenizer, and genomic DNA was extracted using the AllPrep DNA/RNA Mini Kit (Qiagen) according to the manufacturer's instructions. DNA was quantified using a spectrophotometer and stored at $-80^{\circ} \mathrm{C}$. The $5 \mathrm{mC}$ and $5 \mathrm{hmC}$ content of extracted DNA was measured by Methylflash Methylated and Hydroxymethylated DNA Quantification Kits (Colorimetric), respectively, following the manufacturer's instructions (Epigentek, Farmingdale, NY). The methylated and hydroxymethylated fraction of DNA was quantified by reading the absorbance at $450 \mathrm{~nm}$ on a SpectraMax M2 multi-mode plate reader (Molecular Devices Inc., Sunnyvale, CA). All assays 
were performed in duplicate wells and the values from two or three runs were averaged. Results were expressed as a percentage of $5 \mathrm{mC}$ and $5 \mathrm{hmC}$ calculated according to the manufacturer's instructions.

\subsection{0. $m R N A$ expression profiling using a real-time PCR array to examine neuroinflammatory} changes

Total RNA was extracted from the hippocampus, cerebral cortex, and cerebellum using the Allprep DNA/RNA Mini Kit (Qiagen) according to the manufacturer's instructions and quantified using a spectrophotometer. cDNA was generated from $0.5 \mu \mathrm{g}$ total RNA per array using the $\mathrm{RT}^{2}$ First Strand Kit (Qiagen) and analyzed using the $\mathrm{RT}^{2}$ Profiler Rat Inflammatory Response and Autoimmunity PCR Array (Qiagen) by means of a Bio-Rad iCycler real-time PCR Detection System (Bio-Rad Laboratories) per manufacturer instructions. This PCR array analyzes 84 different genes known to be involved in inflammatory and autoimmune responses (including inflammatory cytokines, chemokines, and their receptors) and includes RNA quality controls and internal housekeeping genes to normalize the data for the amount of RNA added to each reverse transcription reaction. Expression profiles were determined using RNA purified from a single rat per array, and arrays were repeated with 6-8 different rats in each experimental group for the hippocampus, cortex, and cerebellum. Raw threshold cycle $\left(\mathrm{C}_{\mathrm{T}}\right)$ values were normalized to a panel of 5 housekeeping genes on the array using software from Qiagen, and the analysis tool was used to perform relative quantification using the $\Delta \Delta \mathrm{C}_{\mathrm{T}}$ method to reveal statistically significant fold differences. Differentially expressed mRNAs were considered significant using a cutoff value $>2.5$ fold change and $p<0.01$. A complete list of genes contained in the array can be viewed on the following link: http://www.sabiosciences.com/rt_pcr_product/HTML/PARN-077A.html.

\subsection{Measurement of proinflammatory cytokine protein}

The quantity of rat tumor necrosis factor alpha (TNF- $\alpha$; Life Technologies Corp.), interleukin 1 beta (IL-1 $\beta$; R\&D Systems, Minneapolis, MN), and IL-6 (Life Technologies Corp.) was determined in brain lysates from 7 GWI rats and 8 vehicle control rats by enzyme-linked immunosorbent assay kits according to the manufacturers' protocols. Briefly, hippocampus, cerebral cortex, and cerebellum samples were homogenized in ice cold phosphate buffered saline 
(pH 7.4) containing a protease inhibitor cocktail (RPI Corp., Mt. Prospect, IL). After homogenization, lysates were centrifuged 10 minutes at $10,000 \times \mathrm{g}(13,000 \mathrm{rpm})$ at $4^{\circ} \mathrm{C}$ and the supernatants were stored at $-80^{\circ} \mathrm{C}$. Protein concentration was determined using the bicinchoninic acid assay (Pierce BCA Protein Assay Kit, Thermo Fisher Scientific, Waltham, MA) and the volume of each sample was normalized to deliver the same amount of total protein per assay (500 $\mu \mathrm{g} / \mathrm{ml}$ for TNF- $\alpha, 400 \mu \mathrm{g} / \mathrm{ml}$ for IL-1 $\beta$, and $250 \mu \mathrm{g} / \mathrm{ml}$ for IL-6). The detection limit was 4 $\mathrm{pg} / \mathrm{ml}$ for TNF- $\alpha, 5 \mathrm{pg} / \mathrm{ml}$ for IL- $1 \beta$, and $5 \mathrm{pg} / \mathrm{ml}$ for IL-6. All assays were performed in duplicate wells. Concentrations were calculated relative to the appropriate standard curve and expressed as $\mathrm{pg} / \mathrm{mg}$ protein.

\subsection{Histopathological analyses}

Coronal sections (5 $\mu \mathrm{m}$ thick) through the dorsal hippocampus, overlying cerebral cortex, and cerebellum from 7 GWI rats and 6 vehicle control rats were cut using a microtome (Leica Biosystems Inc., Buffalo Grove, IL). Following the manufacturers' protocols, serial sections were stained with $0.002 \%$ Fluoro-Jade B (EMD Millipore Corp., Billerica, MA) to evaluate the presence of degenerating neurons and underwent in situ detection of fragmented DNA [terminal deoxynucleotidyl transferase dUTP nick end lableling (TUNEL) assay using the TACS 2 TdT DAB Kit, Trevigen, Gaithersburg, MD] to examine apoptosis. Positive control sections for Fluoro-Jade B staining were obtained from FD NeuroTechnologies Inc. (Columbia, MD). Digital images were captured using cellSens software and an Olympus BX53 microscope (Olympus America Inc., Center Valley, PA).

Ionized calcium binding adapter molecule 1 (Ibal) immunohistochemistry. To examine microglial morphology, sections were deparaffinized and subjected to heat mediated antigen retrieval using Citrate Target Retrieval Solution, pH 6 (Dako North America Inc., Carpinteria, CA). Sections were blocked with $3 \%$ hydrogen peroxide in water for 10 minutes and 5\% normal rabbit serum for 30 minutes at room temperature before incubation overnight at $4^{\circ} \mathrm{C}$ with a goat anti-Iba1 primary antibody (ab5076, Abcam, Cambridge, MA) at 1:1000 dilution. After rinsing, slides were incubated for 1 hour with a secondary antibody (rabbit anti-goat IgG conjugated to biotin) using the Vectastain Elite ABC Kit (Goat IgG) (Vector Laboratories Inc., Burlingame, CA) following the manufacturer's instructions. Sections were then incubated with a 3,3'- 
diaminobenzidine (DAB) chromogen-substrate to form a colored reaction product, counterstained with Mayer's hematoxylin (Dako North America Inc.), dehydrated, and permanently mounted. Negative controls were achieved by applying $1 \%$ normal rabbit serum instead of the primary antibody.

CD68 (ED1) immunohistochemistry. To examine microglial activation, deparaffinized sections were subjected to heat mediated antigen retrieval using Target Retrieval Solution, $\mathrm{pH} 9$ (Dako North America Inc.). Sections were blocked with 3\% hydrogen peroxide in methanol for 10 minutes and $10 \%$ normal goat serum for 30 minutes at room temperature before incubation overnight at $4^{\circ} \mathrm{C}$ with a mouse monoclonal anti-CD68 (clone ED1) primary antibody (MCA341R, AbD Serotec, Raleigh, NC) at 1:100 dilution. After rinsing, slides were incubated for 30 minutes with the mouse antibody enhancer and 30 minutes with the polymer-horseradish peroxidase (HRP) for mouse antibody using the Polink-2 Plus HRP Polymer Detection Kit (for mouse antibody on rat tissue; Golden Bridge International Inc., Bothell, WA) following the manufacturer's instructions. Sections were incubated with a DAB chromogen-substrate, counterstained with Mayer's hematoxylin, dehydrated, and permanently mounted. Negative controls were achieved by applying 5\% normal goat serum instead of the primary antibody.

Quantitation of ED1-, TUNEL-, and Fluoro-Jade B-positive cells. The number of ED1positive cells was determined manually in one section of dorsal hippocampus, cortex, and cerebellum per animal by an investigator blinded to treatment. ED1-labeled cells were counted in every third high powered field (HPF, 400× magnification) in the hippocampus and cortex and in every tenth HPF along the granular and Purkinje layers of the cerebellum (equaling $20 \mathrm{HPFs}$ counted in the hippocampus, 16 HPFs counted in the cortex, and 9-16 HPFs counted in the cerebellum per animal). The total number of TUNEL-positive and Fluoro-Jade B-positive neurons was counted throughout 2 sections of hippocampus and cortex and one section of cerebellum per animal. Mean values for each brain region were calculated separately for every animal before the means and standard errors were determined for the total number of animals included per group.

\subsection{Statistical analysis}


Results were reported as means \pm standard error of the mean for vehicle-treated animals and those exposed to a combination of chemicals and stress. Changes in miRNA and mRNA expression analyzed using the miScript Rat Neurological Development and Disease miRNA PCR Array and $\mathrm{RT}^{2}$ Profiler Rat Inflammatory Response and Autoimmunity PCR Array, respectively, were calculated using software from Qiagen. Plasma exosome RNA-seq analysis was performed by Maverix Biomics. All other statistical analyses were performed using SigmaPlot 11.2 software (Systat Software, Inc.) with $\mathrm{p}<0.05$ considered significant. Differences in molecular, cytologic, and histologic parameters between exposed and control groups were evaluated using unpaired t tests. The Pearson Product Moment Correlation was used to examine the relationship between global $5 \mathrm{hmC}$ and $5 \mathrm{mC}$ content. Nonparametric tests were used when indicated. Statistical outliers ( $\geq$ two standard deviations from the mean) were removed from the analyses, which included one control rat identified during evaluation of ED1 staining.

\section{Results}

\subsection{General health observations}

A total of 65 rats ( $n=33$ GWI and $n=32$ vehicle control rats) were used in this study to evaluate the chronic, delayed effects of GW-relevant exposures. Over the course of the one-year post-exposure period, $9 \%$ of rats ( 3 GWI, 3 controls) died prior to the completion of the study due to age-related illnesses which appeared to be unrelated to GW exposures. These animals were used for body weight comparisons during the exposure regimen but were eliminated from all other analyses. The general behavior and clinical signs of health were similar between groups, with no visible signs of neurotoxicity, pain, or discomfort. Nevertheless, GWI rats demonstrated reduced weight gain relative to controls during the 4-week exposure period (Fig. 1). This difference in weight gain was eliminated by two weeks after completion of the exposure regimen $(p>0.05)$ and weights were similar throughout the year-long study period.

\subsection{Altered miRNA expression in the brain one year after $G W$-related exposures}

Using a cutoff value $>2.5$ fold change and $\mathrm{p}<0.01$, results from the Rat Neurological Development and Disease miRNA PCR array performed on 7-9 different rats in each experimental group revealed that 6 miRNAs were differentially expressed in the hippocampus 
including rat miR-124-3p, miR-29b-3p, miR-22-3p, miR-33-5p, miR-381-3p, and miR-488-3p (Fig. 2). In contrast, no miRNAs were altered in the cortex or cerebellum using the same cutoff value. When quantitative real-time RT-PCR analysis was performed on $9 \mathrm{GWI}$ and 9 control hippocampal RNA samples (re-extracted from the contralateral side used in the array) using primer sets for these 6 miRNAs from Qiagen, differential expression was confirmed only for miR-124-3p (1.73 fold upregulation in GWI, $\mathrm{p}=0.02$ ) and miR-29b-3p (1.56 fold upregulation in GWI, $p=0.001$ ). Levels of miR-124-3p and miR-29b-3p were abundant in the three brain regions examined (miR-124-3p ranged from 8-fold to 45-fold higher and miR-29b-3p ranged from 3-fold to 12-fold higher than that of housekeeping small noncoding RNAs on the PCR array; Table 1; Fig. 2). While the most significant fold increase in these two miRNAs was observed in the hippocampus of GWI rats, these miRNAs also exhibited an elevation in the cortex and cerebellum that did not reach statistical significance as detected by the PCR array (Table 1).

\subsection{Evaluation of validated and predicted target $m R N A s$}

Because mature miR-124 and miR-29b are wholly homologous between rats and humans, target prediction was determined separately for both rat and human species (Jiao et al., 2010; Pan-Vazquez et al., 2015). Evaluation of rat putative target mRNAs using miRecords identified 461 validated and predicted targets of the differentially expressed miRNAs (rno-miR-124 and rno-miR-29b) observed in the hippocampus of GWI rats. When the search in miRecords was repeated to evaluate human putative targets of the differentially expressed miRNAs (hsa-miR124 and hsa-miR-29b) using more stringent criteria (i.e., agreement from $\geq 6 / 11$ target prediction programs for human compared to $\geq 3 / 11$ for rat), 362 validated and predicted targets were identified. To gain functional insights into the miRNAs altered in GWI rats, gene sets were constructed corresponding to biological pathways and networks underlying complex diseases using the online tool DAVID. Five pathways were matched with rat gene sets that remained significant after the adjustment for multiple hypothesis testing using the conservative Benjamini correction; likewise, 6 pathways were matched with human gene sets (Table 2). The most highly enriched pathways that overlapped in both rat and human searches include extracellular matrix (ECM)-receptor interaction, focal adhesion, and the integrin signaling pathway. Other significant pathways include the cadherin signaling pathway, the Wnt signaling pathway, and axon guidance. 


\subsection{Differentially expressed hippocampal miRNAs as potential noninvasive biomarkers of GWI}

To determine whether miR-124-3p and miR-29b-3p could serve as potentially useful biomarkers in circulation, quantitative real-time RT-PCR analysis was performed using RNA extracted from individual plasma samples from $30 \mathrm{GWI}$ and 27 control rats. Neither miRNA showed differences in expression levels between experimental groups. In contrast to the high levels of miR-124-3p found in the brain, very low levels were detected in plasma $\left(\mathrm{C}_{\mathrm{T}}\right.$ values were approximately 31). Higher levels of miR-29b-3p were detectable in plasma compared to miR-124-3p ( $\mathrm{C}_{\mathrm{T}}$ values for miR-29b-3p were approximately 25$)$ and good recovery was observed with the C. elegans miR-39 miRNA mimic $\left(\mathrm{C}_{\mathrm{T}}\right.$ values were approximately 21$)$.

\subsection{Plasma exosome RNA-seq analysis}

Because circulating miR-124-3p and miR-29b-3p did not exhibit diagnostic potential in this established rat model of GWI, we performed RNA-seq analysis of exosome RNAs isolated from pooled plasma samples from $29 \mathrm{GWI}$ rats and 24 control rats as a first step to expand the search for additional miRNAs and other small noncoding RNAs that could serve as potential novel biomarkers of GWI. The small RNA yield from each of the pooled GWI and control plasma samples was $19 \mathrm{ng}$ and $22 \mathrm{ng}$ per $500 \mu \mathrm{l}$ plasma, respectively, which is consistent with RNA yields in human plasma samples also determined using an Agilent Bioanalyzer (Huang et al., 2013). Exosome RNA sizes ranging from approximately 20 nucleotides to approximately 150 nucleotides in length were used for library generation. For the GWI exosome library, the number of raw single-end reads retained after quality assessment used for mapping was about 17 million, while the number of retained raw reads for the control exosome library was about 8.5 million. Although quality assessment determined that the quality of the sequences appeared good, only about $10 \%$ of reads for the GWI sample and $14 \%$ of reads for the control sample mapped to the rat genome (the number of aligned reads was approximately 1.7 million and 1.2 million for the GWI and control samples, respectively).

Exosome RNA-seq analysis demonstrated that the circulating rat exosomes contain a broad range of RNA species. RNA species that mapped to the rat genome included noncoding RNAs [miRNAs, tRNAs, piwi-interacting RNAs (piRNAs), long noncoding RNAs (lncRNAs), and other noncoding RNAs], coding sequences, and antisense transcripts (Fig. 3). A total of 531 
known miRNAs were detected in the two pooled samples although no statistically significant differences in miRNA expression were identified between the GWI and control groups. The top 20 most abundant miRNAs made up $67.3 \%$ of all mappable exosome miRNAs and are presented in Table 3. Despite the fact that our investigation did not include replicates (i.e., only one pooled GWI plasma sample and one pooled control sample were sequenced) making it challenging to detect significant differences, analysis nevertheless determined that GW exposures induced the differential expression ( $\log 2$ fold change, $\mathrm{p}<0.05)$ of two rat exosomal piRNAs in circulation. These included rno-piR-007899 (8.2 fold upregulation in GWI, p=0.004) and rno-piR-019162 (7.2 fold downregulation in GWI, $\mathrm{p}=0.02$ ).

\subsection{DNA methylation and hydroxymethylation status}

Quantification of global 5mC and 5hmC content of extracted DNA from 7 GWI rats and 8 control rats revealed alterations in DNA methylation and hydroxymethylation one year after GW-related exposures (Fig. 4). A 46\% increase in 5mC content was detected in the hippocampus, with no changes in the cortex or cerebellum. A $45 \%$ decrease in $5 \mathrm{hmC}$ content in the cortex and a $29 \%$ increase in the cerebellum was also identified. In control animals, levels of $5 \mathrm{mC}$ and $5 \mathrm{hmC}$ were positively correlated in the hippocampus $(\mathrm{r}=0.84, \mathrm{p}=0.009)$, cortex $(\mathrm{r}=0.94$, $\mathrm{p}=0.0006)$, and cerebellum $(\mathrm{r}=0.89, \mathrm{p}=0.003)$. The relationship between $5 \mathrm{mC}$ and $5 \mathrm{hmC}$ was weakened in GWI animals in the hippocampus $(\mathrm{r}=0.77, \mathrm{p}=0.04)$ and cerebellum $(\mathrm{r}=0.78$, $\mathrm{p}=0.04)$, and eliminated in the cortex $(\mathrm{r}=0.51, \mathrm{p}=0.25)$.

\subsection{Neuroinflammatory and neurodegenerative changes}

Overall, the results from the mRNA, protein, and histopathological analyses demonstrated little to no evidence of neuroinflammatory changes detected one year after exposure to a combination of PB, DEET, permethrin, and stress. Using a cutoff value $>2.5$ fold change and $\mathrm{p}<0.01$, results from the Rat Inflammatory Response and Autoimmunity PCR array performed on 6-8 different rats in each experimental group revealed no differentially expressed mRNAs in the hippocampus or cortex. Six of 84 mRNAs examined were altered in the cerebellum including Cc112, Ccl2, Cxc12, Il17a, Kng1, and Nfkb1 (Supplementary Table 1). In the hippocampus, cortex, and cerebellum, no differences in the levels of IL-1 $\beta$ or IL-6 protein were observed between GWI $(n=7)$ and control $(n=8)$ rats (Table 4). TNF- $\alpha$ levels were not different in the 
hippocampus but were higher in the cortex and lower in the cerebellum of GWI animals. Overall, the levels of the proinflammatory cytokines IL-1 $\beta$ and TNF- $\alpha$ in brain lysates were low or nondetectable, which was consistent with the general lack of significant alterations in mRNA expression of these proinflammatory cytokines (Table 5) and predominance of nonactivated (ramified) microglia viewed histologically (Supplementary Figs. 1 and 2). Qualitative evaluation of Iba1-labeled cells revealed similar ramified morphology in both groups in all three brain regions. No significant differences in the number of activated microglia (ED1-positive cells) were found between GWI rats $(n=7)$ and controls $(n=5)$ in the hippocampus, cortex, or cerebellum (Table 6; Supplementary Figs. 3 and 4). In addition, very few to no degenerating neurons or TUNEL-positive cells were detected in any animal (Supplementary Figs. 5 and 6).

\section{Discussion}

After 25 years, GWI remains a complex, untreatable illness for up to 250,000 veterans of the 1990-1991 Persian Gulf War, with cognitive dysfunction and mood deficits being among the most prevalent and debilitating symptoms. Although the etiology of GWI is unknown, epidemiological investigations and experimental studies using animal models have determined that these impairments are causally related to neurotoxic chemical exposures such as PB, permethrin, and DEET encountered during deployment (RAC 2008, 2014; Steele et al., 2012; Kerr, 2015; White et al., 2016). The current study provides novel evidence that combined exposure to low doses of GW-related chemicals and mild stress for 28 days causes epigenetic modifications in the brain that persist one year after exposure, including increased expression of miR-124-3p and miR-29b-3p in the hippocampus and regional alterations in global DNA methylation and DNA hydroxymethylation. miRNAs and DNA methylation are two important epigenetic mechanisms in the central nervous system that enable long-term and dynamic regulation of neuronal function and are known to play an important role in learning and memory formation, adult neurogenesis, and the onset of neurological disorders (Miller and Sweatt, 2007; Guo et al., 2011; Salta and De Strooper, 2012; Sun et al., 2011; Grayson and Guidotti, 2013; Coppieters et al., 2014; Iyengar et al., 2014). Results from this investigation implicate the involvement of miRNAs and DNA modifications in the pathophysiology of GWI, although further studies are needed to evaluate whether they are causal factors for the health effects of this complex illness or are chronic markers of GW-related exposures. 
An upregulation of miR-124-3p and miR-29b-3p in the hippocampus of GWI rats is consistent with other rodent studies that demonstrated impaired synaptic integrity and reduced hippocampal neurogenesis (thought to be critical for hippocampal plasticity and memory formation) associated with observed memory and mood deficits following GW exposures (Parihar et al., 2013; Ojo et al., 2014; Megahed et al., 2015; Zakirova et al., 2015). miRNAs are known to be enriched in the synapse, are critical regulators of synaptic function, and enable finetuning of expression profiles necessary for activity-dependent synaptic plasticity by silencing target mRNAs at the synapse in a reversible manner (Olde Loohuis et al., 2012; Garza-Manero et al., 2014). Each miRNA can regulate the expression of hundreds of genes posttranscriptionally and can target many mRNAs in different cellular and developmental conditions (Olde Loohuis et al., 2012). Both miR-124 and miR-29b are enriched in the brain and are known to play critical roles in cognition. miR-124 i) inhibits glucocorticoid receptor gene expression thereby affecting a variety of glucocorticoid receptor-mediated physiological processes, ii) regulates signaling molecules underlying synaptic plasticity and memory formation including those in the brainderived neurotrophic factor (BDNF)/cAMP response element-binding protein (CREB) pathway, iii) is involved in neurogenesis, neurodegeneration, synapse morphology, neurodevelopment, neurotransmission, and chronic stress, and iv) is the most abundant miRNA in the brain (Fischbach and Carew, 2009; Vreugdenhil et al., 2009; Mannironi et al., 2013; Pan-Vazquez et al., 2015; Sun et al., 2015). Increased miR-124 has been shown to negatively impact memory in mice by downregulation of $\alpha$-amino-3-hydroxy-5-methyl-4-isoxazolepropionic acid (AMPA)mediated glutamate signaling, while hippocampal miR-124 inhibition has been shown to correlate with enhanced memory performance (Dutta et al., 2013). Interestingly, a polymorphism in the miR-124 target gene IQ motif containing GTPase actvating protein 1 (IQGAP1), which impairs miR-124 binding to IQGAP1, has been shown to improve human cognitive agility (Yang et al., 2014). miR-29b i) targets genes involved in epigenetic regulation pathways including DNA methyltransferases, ii) is a known regulator of synaptic morphology, iii) is upregulated in a methyl $\mathrm{CpG}$ binding protein 2 (MECP2) knockout mouse which is characterized by a loss of cognitive, social, and motor abilities and reduction in dendritic spines, and iv) is a master regulator of a large number of collagen genes and other molecules of the ECM including integrin- $\beta 1$, laminin- $\gamma 1$, fibrillin 1, elastin, and matrix metalloproteinase 2 (Garzon et al., 2009; Liu et al., 2010; Urdinguio et al., 2010). miR-29b has also been shown to decrease the expression 
of human progranulin, which is a secreted glycoprotein whose reduced levels are implicated in frontotemporal dementia (Jiao et al., 2010).

Functional classification of validated and predicted rat and human miR-124 and miR-29b target mRNAs revealed that the most significant and interrelated pathways identified included ECM-receptor interaction, focal adhesion, integrin signaling, cadherin signaling, signaling by PDGF, axon guidance, and Wnt signaling. These pathways are known to be involved in synaptic function, synaptic plasticity, and memory formation and may serve as candidate molecular pathways that may be targeted for the development of novel therapeutics for GWI (Chan et al., 2006; Wu, 2007; Liu et al., 2010; Hunt et al., 2011; Jafari et al., 2012; Warren et al., 2012; Bellot et al., 2014; Friedman et al., 2015; Hu et al., 2015). The focal adhesion pathway has been identified in a human study that performed genome-wide association to identify genes influencing information processing speed, an important cognitive function that is compromised in old age and in neurological disorders such as schizophrenia and depression (Luciano et al., 2011). In addition, the Wnt signaling pathway has been shown to modulate cognitive function in the adult mouse brain and reverse cognitive deficits in an Alzheimer's disease model (Oliva et al., 2013; Vargas et al., 2014).

Recent studies have suggested that circulating miRNAs are potential noninvasive markers for disease assessment (Etheridge et al., 2011). In the rat model of GWI, we did not detect altered miRNAs in plasma as candidate biomarkers of GW-relevant exposure using quantitative RTPCR analysis targeting differentially expressed miRNAs found in the brain (miR-124-3p and miR-29b-3p) or via next generation sequencing analysis of circulating exosome small RNAs. However, we did detect the differential expression of two piRNAs (rno-piR-007899 and rno-piR019162) whose pathophysiological significance is presently unknown. piRNAs have been proposed to play roles in the epigenetic regulation of cancers, stroke, and other diseases, and their discovery in human blood and saliva reveals that they may serve as novel, stable, noninvasive biomarkers for disease detection and monitoring similar to miRNAs (Cui et al., 2011; Dharap et al., 2011; Bahn et al., 2015; Yang et al., 2015). In contrast to miRNAs which are highly conserved across species, piRNAs are typically generated from long genomic clusters which are not necessarily sequence-conserved, but their relative chromosomal locations are conserved, suggesting that their location of origin is critical to their function (Landry et al., 2013). Therefore, while the differentially expressed piRNAs observed in the rat model of GWI 
are unlikely to be identified in humans, evaluation of the diagnostic potential of novel circulating piRNAs via high throughput RNA sequencing of blood or saliva in veterans with GWI is certainly warranted.

The piRNAs are a relatively new, abundant class of small (26-32 nucleotides in length) noncoding RNAs initially thought to be expressed only in germline tissues and to maintain germline genome integrity by repressing transposable elements through de novo DNA methylation (Lee et al., 2011; Iyengar et al., 2014). However, the discovery that a large majority of piRNAs in mammals are derived from non-transposon-associated, gene-rich regions of the genome and that piRNAs are also expressed in somatic cells suggests broader functions for piRNAs beyond transposon silencing, including regulation of gene expression (Landry et al., 2013). Interestingly, increasing evidence has revealed that the brain has elevated transposable element activity relative to other somatic tissues which changes in response to environmental stimuli such as stress and varies by brain region, with the highest rates of transposable element insertions in the hippocampus (Lapp and Hunter, 2016). Transposable elements are known to affect local chromatin states and the expression of nearby genes, and dysregulated transposon activity has been implicated in mental disorders including major depression, bipolar disorder, post-traumatic stress disorder, and schizophrenia (Lapp and Hunter, 2016).

Although the function of many piRNAs is not currently known, recent evidence provides a role for piRNAs in the epigenetic control of memory. Lee et al. identified piRNAs in the mouse hippocampus and suggested that possible targets include genes involved in dendritic spine morphogenesis (Lee et al., 2011). Neuronal piRNAs that stably associate with a neuronal Piwi protein were also discovered to be abundantly expressed in the central nervous system of Aplysia and were upregulated in response to serotonin, a neuromodulator important for learning and memory (Rajasethupathy et al., 2012). The authors found that the Piwi/piRNA complex in neurons can methylate target genes involved in memory-related synaptic plasticity (in particular CREB2, a critical plasticity-related gene and transcriptional repressor of memory; Rajasethupathy et al., 2012). It is tempting to speculate that altered piRNA levels detected in GWI rats are indicative of disruption of normal transposon activity and/or long-lasting changes in gene expression of neurons involved in long-term memory storage. Studies investigating changes in brain piRNA profiles in animal models of GWI are needed to elucidate the possible contribution of altered piRNAs to GWI pathophysiology. 
In addition to noncoding RNAs, epigenetic modifications such as DNA methylation are known to play a role in normal and pathological brain functioning. Extensive active DNA modifications have been observed in mature neurons in vivo, including DNA demethylation and de novo methylation (Guo et al., 2011). Activity-modified DNA methylation is enriched in genes involved in synaptic function, neuronal differentiation, protein phosphorylation, and the calcium signaling pathway (Guo et al., 2011). DNA methylation involves the addition of a methyl group to the 5 position of cytosine which is catalyzed by DNA methyltransferases to form $5 \mathrm{mC}$, and DNA hydroxymethylation occurs when methyl groups on DNA are oxidized to 5-hydroxymethyl groups by the ten-eleven-translocation family of enzymes forming $5 \mathrm{hmC} .5 \mathrm{mC}$ is generally associated with inhibition of gene expression, while $5 \mathrm{hmC}$, whose levels are found to be highest in the brain, is generally associated with increased expression (Miller and Sweatt, 2007; Guo et al., 2011; Chen et al., 2012; Wen and Tang, 2014). As a first step to evaluate whether altered DNA methylation dynamics underlie the pathogenesis of GWI, global 5mC and $5 \mathrm{hmC}$ content of extracted DNA was quantified in the hippocampus, cortex, and cerebellum one year after exposure to GW agents. Brain region-specific differences were observed in GWI animals in which $5 \mathrm{mC}$ content was significantly increased in the hippocampus, whereas $5 \mathrm{hmC}$ content was significantly decreased in the cortex but increased in the cerebellum one year after exposure. Additional studies are needed to determine whether altered methylation status following GWrelevant exposures occurs in the promoters and other regulatory elements of genes critical for neuronal function.

Results from this study determined that the persistent epigenetic changes observed one year after exposure to a combination of $\mathrm{PB}$, DEET, permethrin, and mild stress occurred in the absence of underlying neuroinflammation and neuronal damage. These observations are consistent with other rodent studies evaluating exposure to various combinations of PB, DEET, and permethrin that showed no histopathological evidence of microglial activation or deterioration of neurons in the hippocampus and cortex (Abdullah et al., 2011, 2012; Ojo et al., 2014; Zakirova et al., 2015). Further supporting these results, a recent study by O'Callaghan et al. demonstrated that PB and DEET alone did not produce a neuroinflammatory response across multiple brain regions and showed a lack of remarkable changes in indicators of neurodegeneration, astrogliosis, and microglial activation (O'Callaghan et al., 2015). Despite a lack of neuroinflammation elicited by PB and DEET alone, O'Callaghan et al. did observe 
elevated proinflammatory cytokine and chemokine expression after exposure to diisopropyl fluorophosphate, a sarin surrogate and irreversible acetylcholinesterase inhibitor, which may explain discrepancies among experimental studies incorporating or excluding organophosphate nerve agents (O'Callaghan et al., 2015). In contrast, using the same 28-day exposure regimen as that utilized in the current study, Parihar et al. described mild hippocampal inflammation as assessed by a marginal increase in activated microglia and hypertrophy of astrocytes, and partial loss of hippocampal neurons as determined by stereological quantification of NeuN-positive neurons, which was not attempted in our study (Parihar et al., 2013). Conflicting results also occurred in previous studies by Abdel-Rahman et al. in which the authors reported significant neuronal cell death in multiple brain regions, although it has been argued that the neurodegeneration interpreted in these studies is actually histological artifact (Abdel-Rahman et al., 2002, 2004; Jortner, 2006).

\section{Conclusion}

Findings from this study implicate a role for epigenetic changes in the pathogenesis of GWI and provide insight into molecular mechanisms and biological pathways that can be targeted for the development of novel treatments for this chronic, debilitating illness. Investigation of the involvement of epigenetic processes including DNA methylation/hydroxymethylation, RNAbased mechanisms, and other processes not addressed in this study such as histone posttranslational modifications in veterans with GWI is needed. These results also may have broader implications for people increasingly exposed to pesticide mixtures.

\section{Acknowledgements}

This work was funded by Department of Defense Congressionally Directed Medical Research Programs (CDMRP) Gulf War Illness Research Program (GWIRP) grant award FMBB100528621 (GW120033) to LMP. CDMRP had no involvement in the study design, collection, analysis and interpretation of data, or writing of the manuscript. 


\section{References}

Abdel-Rahman A, Shetty AK, Abou-Donia MB. Disruption of the blood-brain barrier and neuronal cell death in cingulate cortex, dentate gyrus, thalamus, and hypothalamus in a rat model of Gulf-War Syndrome. Neurobiol Dis 2002;10:306-326.

Abdel-Rahman A, Abou-Donia S, El-Masry E, Shetty A, Abou-Donia M. Stress and combined exposure to low doses of pyridostigmine bromide, DEET, and permethrin produces neurochemical and neuropathological alterations in cerebral cortex, hippocampus, and cerebellum. J Toxicol Environ Health A 2004;67:163-192.

Abdullah L, Crynen G, Reed J, et al. Proteomic CNS profile of delayed cognitive impairment in mice exposed to Gulf War agents. Neuromolecular Med 2011;13:275-288.

Abdullah L, Evans JE, Bishop A, et al. Lipidomic profiling of phosphocholine-containing brain lipids in mice with sensorimotor deficits and anxiety-like features after exposure to Gulf War agents. Neuromolecular Med 2012;14:349-361.

Bahn JH, Zhang Q, Li F, et al. The landscape of microRNA, Piwi-interacting RNA, and circular RNA in human saliva. Clin Chem 2015;61:221-230.

Bellot A, Guivernau B, Tajes M, Bosch-Morató M, Valls-Comamala V, Muñoz FJ. The structure and function of actin cytoskeleton in mature glutamatergic dendritic spines. Brain Res 2014;1573:1-16.

Chan CS, Weeber EJ, Zong L, Fuchs E, Sweatt JD, Davis RL. Beta 1-integrins are required for hippocampal AMPA receptor-dependent synaptic transmission, synaptic plasticity, and working memory. J Neurosci 2006;26:223-232.

Chao LL, Abadjian L, Hlavin J, Meyerhoff DJ, Weiner MW. Effects of low-level sarin and cyclosarin exposure and Gulf War Illness on brain structure and function: a study at 4T. Neurotoxicology 2011;32:814-822.

Chen H, Dzitoyeva S, Manev H. Effect of aging on 5-hydroxymethylcytosine in the mouse hippocampus. Restor Neurol Neurosci 2012;30:237-245.

Collotta M, Bertazzi PA, Bollati V. Epigenetics and pesticides. Toxicology 2013;307:35-41.

Coppieters N, Dieriks BV, Lill C, Faull RL, Curtis MA, Dragunow M. Global changes in DNA methylation and hydroxymethylation in Alzheimer's disease human brain. Neurobiol Aging 2014;35:1334-1344.

Corbel V, Stankiewicz M, Pennetier C, et al. Evidence for inhibition of cholinesterases in insect and mammalian nervous systems by the insect repellent deet. BMC Biol. 2009;7:47.

Cui L, Lou Y, Zhang X, Zhou H, Deng H, Song H, Yu X, Xiao B, Wang W, Guo J. Detection of circulating tumor cells in peripheral blood from patients with gastric cancer using piRNAs as markers. Clin Biochem 2011;44:1050-1057. 
Dharap A, Nakka VP, Vemuganti R. Altered expression of piRNA in rat brain following transient focal ischemia. Stroke 2011;42:1105-1109.

Dutta R, Chomyk AM, Chang A, et al. Hippocampal demyelination and memory dysfunction are associated with increased levels of the neuronal microRNA miR-124 and reduced AMPA receptors. Ann Neurol 2013;73:637-645.

Etheridge A, Lee I, Hood L, Galas D, Wang K. Extracellular microRNA: a new source of biomarkers. Mutat Res 2011;717:85-90.

Fischbach SJ, Carew TJ. MicroRNAs in memory processing. Neuron 2009;63:714-716.

Friedman A, Kaufer D, Shemer J, Hendler I, Soreq H, Tur-Kaspa I. Pyridostigmine brain penetration under stress enhances neuronal excitability and induces early immediate transcriptional response. Nat Med 1996;2:1382-1385.

Friedman LG, Benson DL, Huntley GW. Cadherin-based transsynaptic networks in establishing and modifying neural connectivity. Curr Top Dev Biol 2015;112:415-465.

Garza-Manero S, Pichardo-Casas I, Arias C, Vaca L, Zepeda A. Selective distribution and dynamic modulation of miRNAs in the synapse and its possible role in Alzheimer's Disease. Brain Res 2014;1584:80-93.

Garzon R, Liu S, Fabbri M, et al. MicroRNA-29b induces global DNA hypomethylation and tumor suppressor gene reexpression in acute myeloid leukemia by targeting directly DNMT3A and 3B and indirectly DNMT1. Blood 2009;113:6411-6418.

Grayson DR, Guidotti A. The dynamics of DNA methylation in schizophrenia and related psychiatric disorders. Neuropsychopharmacology 2013;38:138-166.

Guo JU, Ma DK, Mo H, et al. Neuronal activity modifies the DNA methylation landscape in the adult brain. Nat Neurosci 2011;14:1345-1351.

Hattiangady B, Mishra V, Kodali M, Shuai B, Rao X, Shetty AK. Object location and object recognition memory impairments, motivation deficits and depression in a model of Gulf War Illness. Front Behav Neurosci 2014;8:78.

Heffner TG, Hartman JA, Seiden LS. A rapid method for the regional dissection of the rat brain. Pharmacol Biochem Behav 1980;13:453-456.

$\mathrm{Hu}$ W, An C, Chen WJ. Molecular mechanoneurobiology: an emerging angle to explore neural synaptic functions. Biomed Res Int 2015;2015:486827.

Huang DW, Sherman BT, Lempicki RA. Systematic and integrative analysis of large gene lists using DAVID Bioinformatics Resources. Nature Protoc 2009a;4:44-57.

Huang DW, Sherman BT, Lempicki RA. Bioinformatics enrichment tools: paths toward the comprehensive functional analysis of large gene lists. Nucleic Acids Res. 2009b;37:1-13. 
Huang X, Yuan T, Tschannen M, et al. Characterization of human plasma-derived exosomal RNAs by deep sequencing. BMC Genomics 2013;14:319.

Hubbard NA, Hutchison JL, Motes MA, et al. Central executive dysfunction and deferred prefrontal processing in veterans with Gulf War Illness. Clin Psychol Sci 2014;2:319-327.

Hunt S, Jones AV, Hinsley EE, Whawell SA, Lambert DW. MicroRNA-124 suppresses oral squamous cell carcinoma motility by targeting ITGB1. FEBS Lett 2011;585:187-192.

Institute of Medicine. Health Consequences of Service During the Persian Gulf War: Recommendations for Research and Information Systems. National Academy Press, Washington, DC, 1996.

Iyengar BR, Choudhary A, Sarangdhar MA, Venkatesh KV, Gadgil CJ, Pillai B. Non-coding RNA interact to regulate neuronal development and function. Front Cell Neurosci 2014;8:47.

Jafari M, Seese RR, Babayan AH, Gall CM, Lauterborn JC. Glucocorticoid receptors are localized to dendritic spines and influence local actin signaling. Mol Neurobiol 2012;46:304315.

Januar V, Ancelin ML, Ritchie K, Saffery R, Ryan J. BDNF promoter methylation and genetic variation in late-life depression. Transl Psychiatry 2015;5:e619.

Jiao J, Herl LD, Farese RV Jr, Gao F-B. MicroRNA-29b regulates the expression level of human progranulin, a secreted glycoprotein implicated in frontotemporal dementia. PLoS ONE 2010;5:e10551.

Jortner BS. The return of the dark neuron. A histological artifact complicating neurotoxicologic evaluation. Neurotoxicology 2006;27:628-634.

Kalani A, Tyagi A, Tyagi N. Exosomes: mediators of neurodegeneration, neuroprotection and therapeutics. Mol Neurobiol 2014;49:590-600.

Kang HK, Li B, Mahan CM, Eisen SA, Engel CC. Health of US veterans of 1991 Gulf War: a follow-up survey in 10 years. J Occup Environ Med 2009;51:401-410.

Kawikova I, Askenase PW. Diagnostic and therapeutic potentials of exosomes in CNS diseases. Brain Res 2015;1617:63-71.

Kerr KJ. Gulf War illness: an overview of events, most prevalent health outcomes, exposures, and clues to pathogenesis. Rev Environ Health 2015;30:273-286.

Landry CD, Kandel ER, Rajasethupathy P. New mechanisms in memory storage: piRNAs and epigenetics. Trends Neurosci 2013;36:535-542.

Lapp HE, Hunter RG. The dynamic genome: transposons and environmental adaptation in the nervous system. Epigenomics 2016;8:237-249. 
Lee EJ, Banerjee S, Zhou H, Jammalamadaka A, Arcila M, Manjunath BS, Kosik KS. Identification of piRNAs in the central nervous system. RNA 2011;17:1090-1099.

Li B, Mahan CM, Kang HK, Eisen SA, Engel CC. Longitudinal health study of US 1991 gulf war veterans: Changes in health status at 10-year follow-up. Am J Epidemiol 2011;174:761-768.

Li X, Spence JS, Buhner DM, et al. Hippocampal dysfunction in Gulf War veterans: investigation with ASL perfusion MR imaging and physostigmine challenge. Radiology 2011;261:218-225.

Liu Y, Taylor NE, Lu L, et al. Renal medullary microRNAs in Dahl salt-sensitive rats: miR-29b regulates several collagens and related genes. Hypertension 2010;55:974-982.

Luciano M, Hansell NK, Lahti J, et al. Whole genome association scan for genetic polymorphisms influencing information processing speed. Biol Psychol 2011;86:193-202.

Manikkam M, Tracey R, Guerrero-Bosagna C, Skinner MK. Pesticide and insect repellent mixture (permethrin and DEET) induces epigenetic transgenerational inheritance of disease and sperm epimutations. Reprod Toxicol 2012;34:708-719.

Mannironi C, Camon J, De Vito F, Biundo A, De Stefano ME, Persiconi I, et al. Acute stress alters amygdala microRNA miR-135a and miR-124 expression: inferences for corticosteroid dependent stress response. PLoSONE 2013;8:e73385.

Megahed T, Hattiangady B, Shuai B, Shetty AK. Parvalbumin and neuropeptide Y expressing hippocampal GABA-ergic inhibitory interneuron numbers decline in a model of Gulf War illness. Front Cell Neurosci 2015;8:447.

Menon PM, Nasrallah HA, Reeves RR, Ali JA. Hippocampal dysfunction in gulf war syndrome. A proton MR spectroscopy study. Brain Res 2004;1009:189-194.

Meyer DA, Carter JM, Johnstone AF, Shafer TJ. Pyrethroid modulation of spontaneous neuronal excitability and neurotransmission in hippocampal neurons in culture. Neurotoxicology 2008;29:213-225.

Mikaelsson MA, Miller CA. The path to epigenetic treatment of memory disorders. Neurobiol Learn Mem 2011;96:13-18.

Miller CA, Sweatt JD. Covalent modification of DNA regulates memory formation. Neuron 2007;53:857-869.

O'Callaghan JP, Kelly KA, Locker AR, Miller DB, Lasley SM. Corticosterone primes the neuroinflammatory response to DFP in mice: potential animal model of Gulf War Illness. J Neurochem 2015;133:708-721.

Odegard TN, Cooper CM, Farris EA, Arduengo J, Bartlett J, Haley R. Memory impairment exhibited by veterans with Gulf War Illness. Neurocase 2013;19:316-327. 
Ojo JO, Abdullah L, Evans J, et al. Exposure to an organophosphate pesticide, individually or in combination with other Gulf War agents, impairs synaptic integrity and neuronal differentiation, and is accompanied by subtle microvascular injury in a mouse model of Gulf War agent exposure. Neuropathology 2014;34:109-127.

Olde Loohuis NF, Kos A, Martens GJ, Van Bokhoven H, Nadif Kasri N, Aschrafi A. MicroRNA networks direct neuronal development and plasticity. Cell Mol Life Sci 2012;69:89-102.

Oliva CA, Vargas JY, Inestrosa NC. Wnts in adult brain: from synaptic plasticity to cognitive deficiencies. Front Cell Neurosci 2013;7:224.

Pan-Vazquez A, Rye N, Ameri M, et al. Impact of voluntary exercise and housing conditions on hippocampal glucocorticoid receptor, miR-124 and anxiety. Mol Brain 2015;8:40.

Parihar VK, Hattiangady B, Shuai B, Shetty AK. Mood and memory deficits in a model of Gulf War Illness are linked with reduced neurogenesis, partial neuron loss, and mild inflammation in the hippocampus. Neuropsychopharmacology 2013;38:2348-2362.

Paxinos G, Watson C. The Rat Brain in Stereotaxic Coordinates. Fourth Edition. Academic Press, San Diego, CA, 1998.

Rajasethupathy P, Antonov I, Sheridan R, Frey S, Sander C, Tuschl T, Kandel ER. A role for neuronal piRNAs in the epigenetic control of memory-related synaptic plasticity. Cell 2012;149:693-707.

Rayhan RU, Stevens BW, Raksit MP, et al. Exercise challenge in Gulf War Illness reveals two subgroups with altered brain structure and function. PLoS One 2013;8:e63903.

Reamon-Beuttner SM, Mutschler V, Borlak J. The next innovation cycle in toxicogenomics: environmental epigenetics. Mutat Res 2008;659:158-165.

Research Advisory Committee on Gulf War Veterans' Illnesses. Gulf War Illness and the Health of Gulf War Veterans. Department of Veterans Affairs, Washington, DC, 2008.

Research Advisory Committee on Gulf War Veterans' Illnesses. Gulf War Illness and the Health of Gulf War Veterans: Research Update and Recommendations, 2009-2013. U.S. Government Printing Office, Boston, MA, 2014.

Revenfeld AL, Bæk R, Nielsen MH, Stensballe A, Varming K, Jørgensen M. Diagnostic and prognostic potential of extracellular vesicles in peripheral blood. Clin Ther 2014;36:830-846.

Ruzicka WB. Epigenetic mechanisms in the pathophysiology of psychotic disorders. Harv Rev Psychiatry 2015;23:212-222.

Salta E, De Strooper B. Non-coding RNAs with essential roles in neurodegenerative disorders. Lancet Neurol 2012;11:189-200. 
Smith BN, Wang JM, Vogt D, Vickers K, King DW, King LA. Gulf war illness: symptomatology among veterans 10 years after deployment. J Occup Environ Med 2013;55:104110.

Snyder JS, Kee N, Wojtowicz JM. Effects of adult neurogenesis on synaptic plasticity in the rat dentate gyrus. J Neurophysiol 2001;85:2423-2431.

Stankiewicz AM, Swiergiel AH, Lisowski P. Epigenetics of stress adaptations in the brain. Brain Res Bull 2013;98:76-92.

Steele L, Sastre A, Gerkovich MM, Cook MR. Complex factors in the etiology of Gulf War Illness: wartime exposures and risk factors in veteran subgroups. Environ Health Perspect 2012;120:112-118.

Sun J, Sun J, Ming G, Song H. Epigenetic regulation of neurogenesis in the adult mammalian brain. Eur J Neurosci 2011;33:1087-1093.

Sun Y, Luo Z-M, Guo X-M, Su D-F, Liu X. An updated role of microRNA-124 in central nervous system disorders: a review. Front Cell Neurosci 2015;9:193.

Tal TL, Tanguay RL. Non-coding RNAs - novel targets in neurotoxicity. Neurotoxicology 2012;33:530-544.

Urdinguio RG, Fernandez AF, Lopez-Nieva P, et al. Disrupted microRNA expression caused by Mecp2 loss in a mouse model of Rett syndrome. Epigenetics 2010;5:656-663.

Vargas JY, Fuenzalida M, Inestrosa NC. In vivo activation of Wnt signaling pathway enhances cognitive function of adult mice and reverses cognitive deficits in an Alzheimer's disease model. J Neurosci 2014;34:2191-2202.

Vialou V, Feng J, Robison AJ, Nestler EJ. Epigenetic mechanisms of depression and antidepressant action. Annu Rev Pharmacol Toxicol 2013;53:59-87.

Vlassov AV, Magdaleno S, Setterquist R, Conrad R. Exosomes: current knowledge of their composition, biological functions, and diagnostic and therapeutic potentials. Biochim Biophys Acta 2012;1820:940-948.

Vreugdenhil E, Verissimo CSL, Mariman R, et al. MicroRNA 18 and 124a down-regulate the glucocorticoid receptor: implications for glucocorticoid responsiveness in the brain. Endocrinology 2009;150:2220-2228.

Vythilingam M, Luckenbaugh DA, Lam T, et al. Smaller head of the hippocampus in gulf warrelated posttraumatic stress disorder. Psychiatry Res 2005;139:89-99.

Warren MS, Bradley WD, Gourley SL, et al. Integrin $\beta 1$ signals through Arg to regulate postnatal dendritic arborization, synapse density, and behavior. J Neurosci 2012;32:2824-2834. 
Wen L, Tang F. Genomic distribution and possible functions of DNA hydroxymethylation in the brain. Genomics 2014;104:341-346.

White RF, Steele L, O'Callaghan JP, et al. Recent research on Gulf War illness and other health problems in veterans of the 1991 Gulf War: Effects of toxicant exposures during deployment. Cortex 2016;74:449-475.

Wu C. Focal adhesion: a focal point in current cell biology and molecular medicine. Cell Adh Migr 2007;1:13-18.

Xiao F, Zuo Z, Cai G, Kang S, Gao X, Li T. miRecords: an integrated resource for microRNAtarget interactions. Nucleic Acids Res 2009;37:D105-D110.

Yang L, Zhang R, Li M, et al. A functional miR-124 binding-site polymorphism in IQGAP1 affects human cognitive performance. PLoS ONE 2014;9:e107065.

Yang X, Cheng Y, Lu Q, Wei J, Yang H, Gu M. Detection of stably expressed piRNAs in human blood. Int J Clin Exp Med 2015;8:13353-13358.

Zakirova Z, Tweed M, Crynen G, et al. Gulf War agent exposure causes impairment of longterm memory formation and neuropathological changes in a mouse model of Gulf War Illness. PLoS ONE 2015;10:e0119579. 


\section{Figure Legends}

Fig. 1. Effect of daily exposure on body weight. Differences in weight gain between GWI rats and controls were eliminated by 2 weeks after completion of the 28-day exposure period (not shown). ${ }^{*} \mathrm{p}<0.05$, $\mathrm{t}$ test.

Fig. 2. Differentially expressed miRNAs $(>2.5$ fold change, $p<0.01)$ in the hippocampus one year after completion of the GW-relevant exposure period. miRNA expression was measured using the Rat Neurological Development and Disease miRNA PCR Array and expressed relative to housekeeping small non-coding RNAs on the array. Fold change is listed above each miRNA. An asterisk (*) indicates miRNAs whose differential expression was confirmed in re-extracted samples by quantitative real-time RT-PCR.

Fig. 3. Plasma exosome small RNA expression summary. Pie charts summarize the number of reads mapping to each annotation type in each pooled sample. Note the abundance of sequences that did not map to the rat genome.

Fig. 4. Global $5 \mathrm{mC}(\mathrm{A})$ and $5 \mathrm{hmC}(\mathrm{B})$ content in the hippocampus, cortex, and cerebellum of rats one year after the 28-day exposure regimen demonstrating alterations in DNA methylation and DNA hydroxymethylation. ${ }^{*} \mathrm{p}<0.05$ compared to corresponding control group, $\mathrm{t}$ test. 


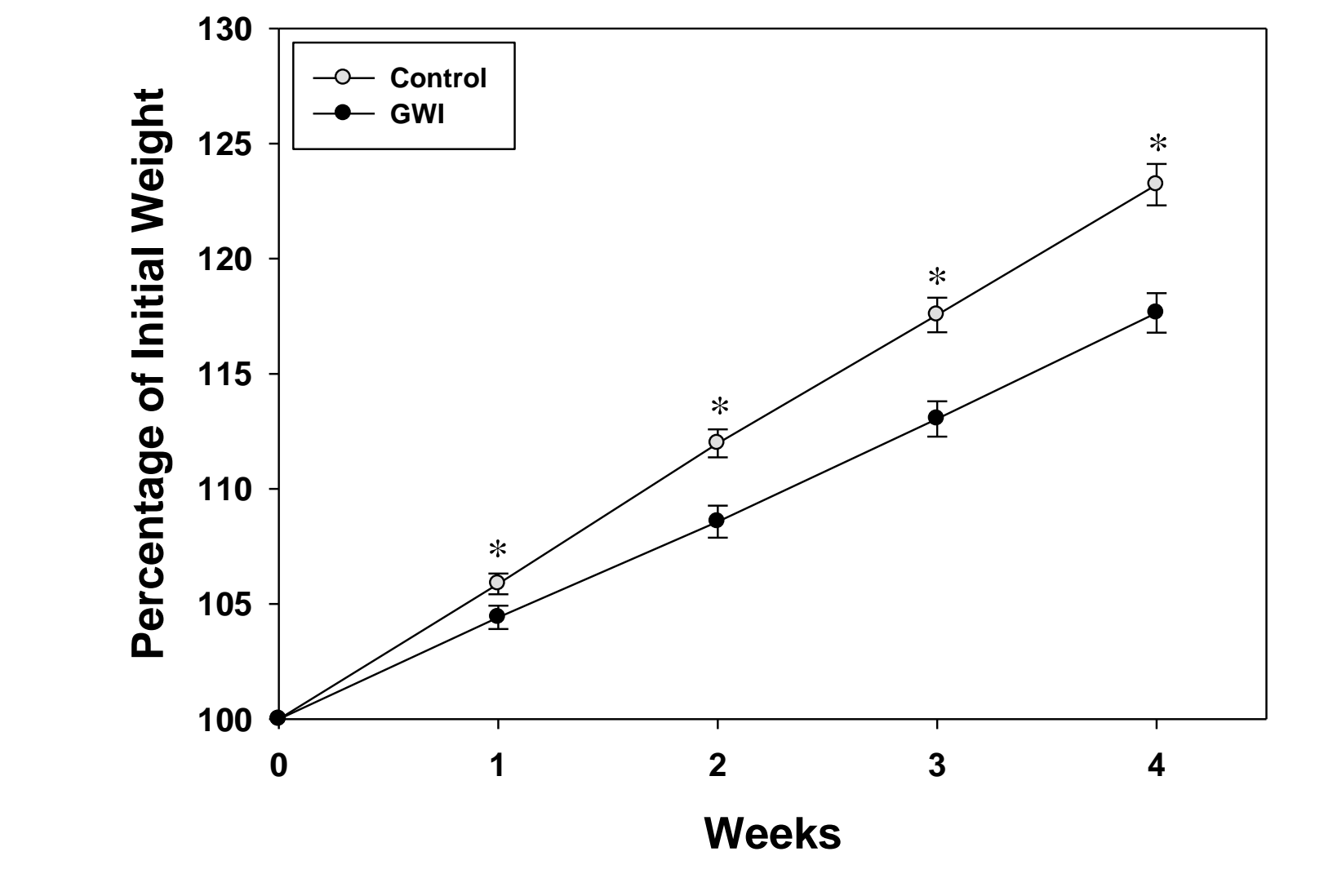

Figure 1

\section{Figure}

Weeks

$$
\text { . }
$$




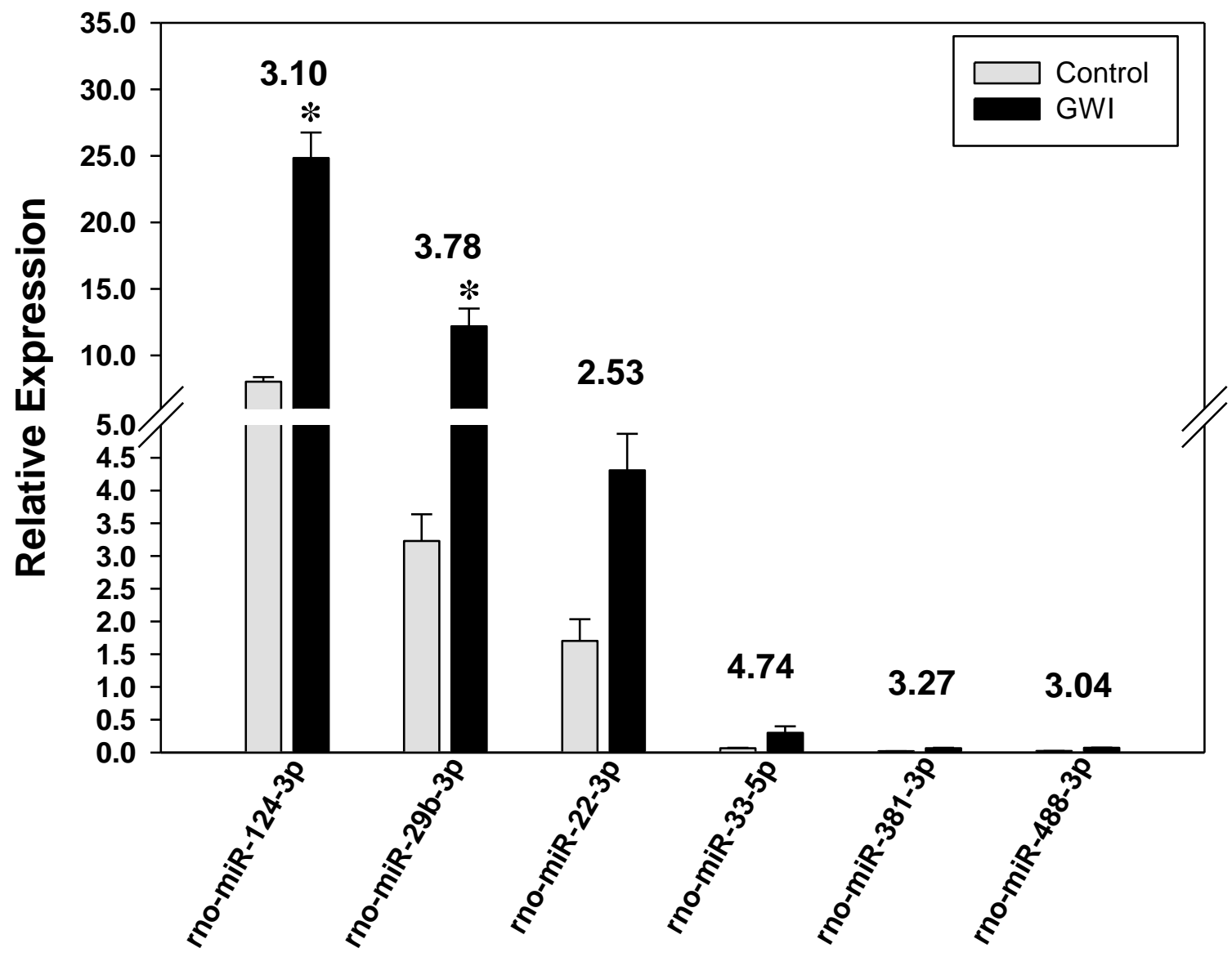


Figure 3

Summary of Reads Mapping to Annotation Types

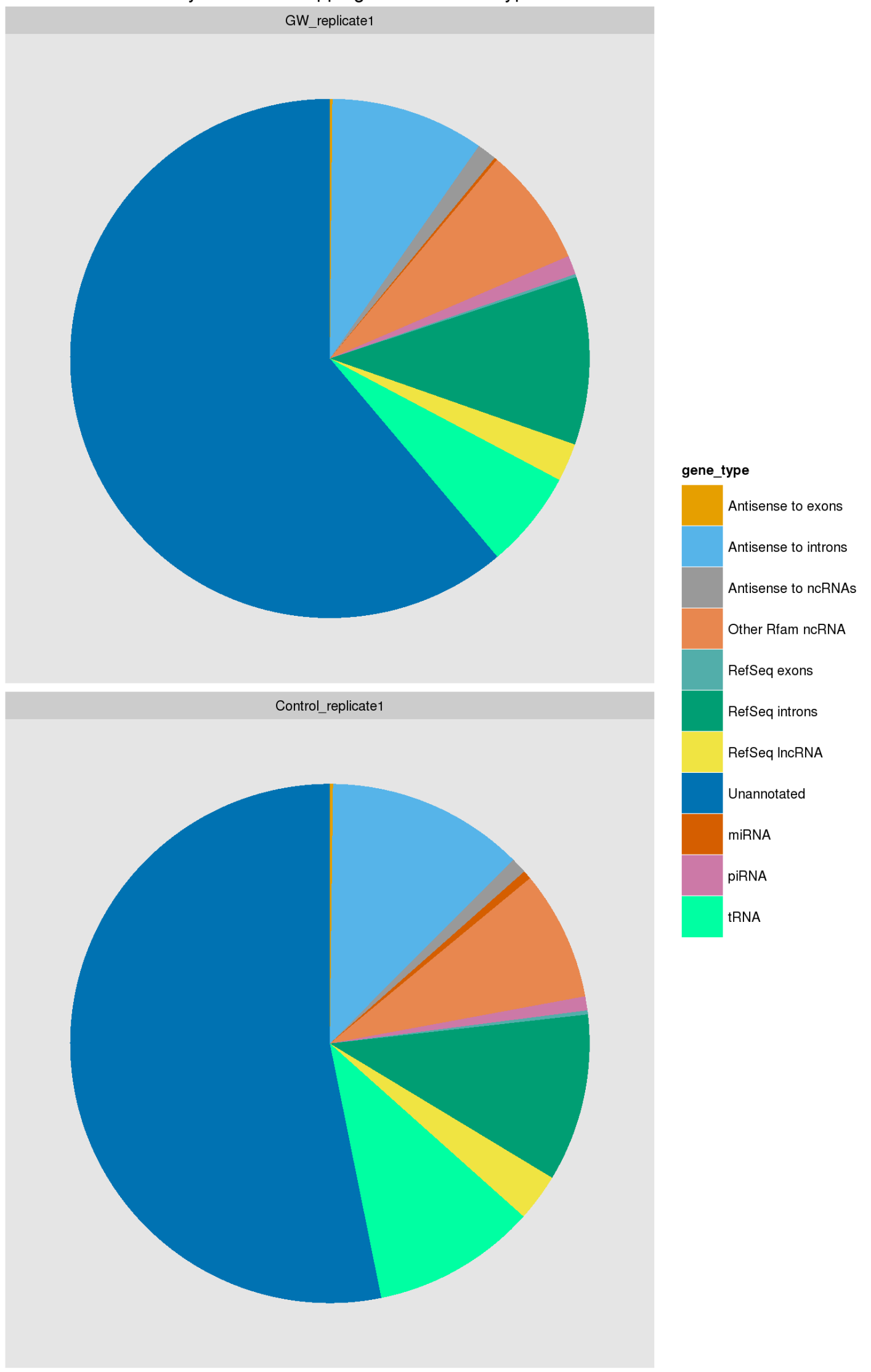


A

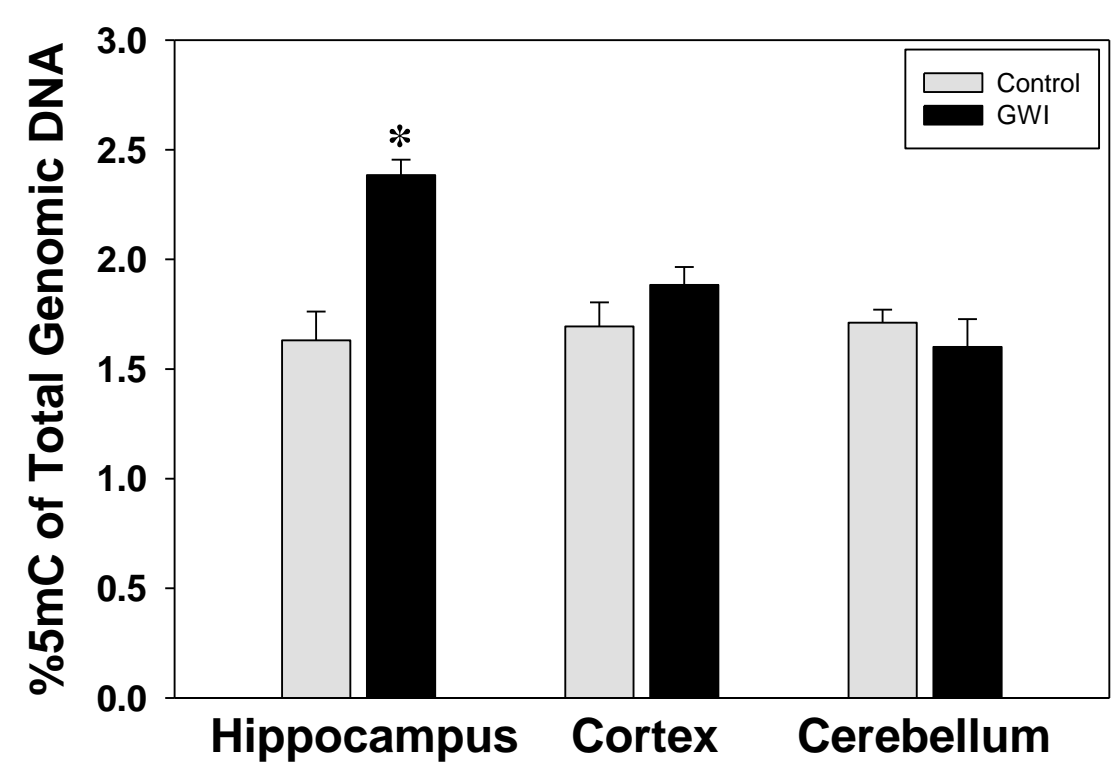

B

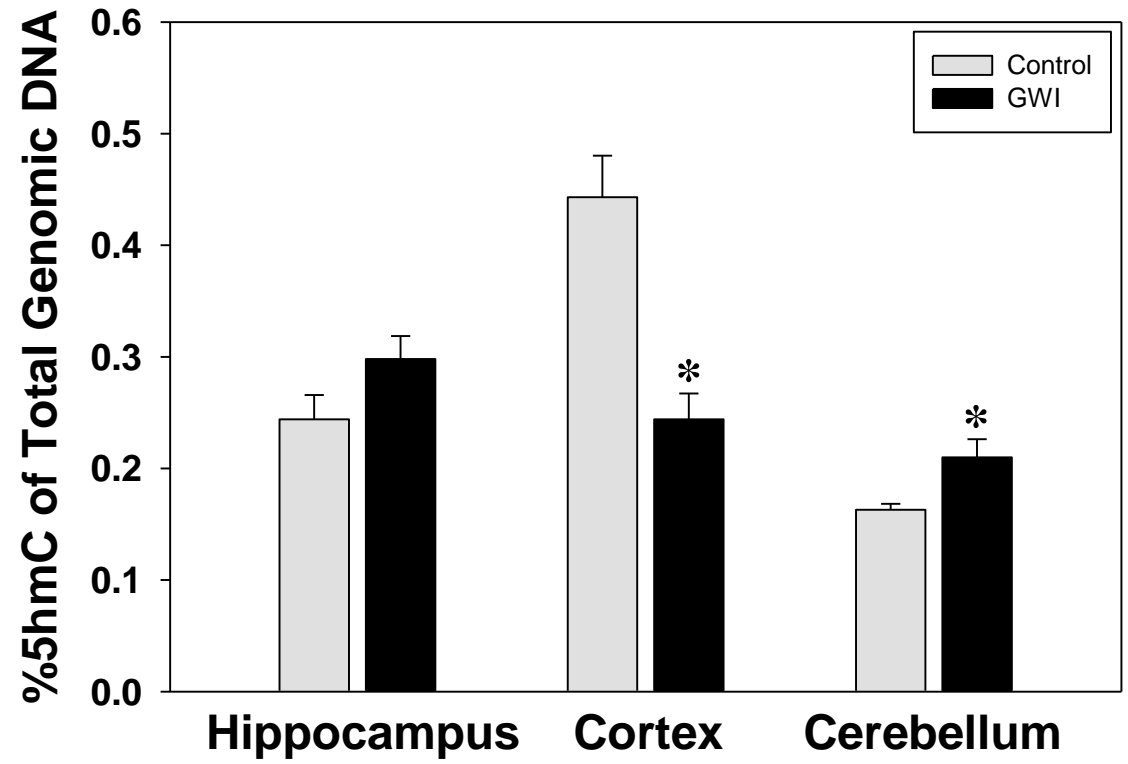


Table 1. Fold expression of miR-124-3p and miR-29b-3p relative to housekeeping small noncoding RNAs (SNORD68 and SNORD96A) one year after exposure to a combination of Gulf War-relevant chemicals and mild stress.

\begin{tabular}{|l|c|c|c|c|c|c|c|l|}
\hline & \multicolumn{4}{|c|}{ miR-124-3p } & \multicolumn{4}{c|}{ miR-29b-3p } \\
\hline & Control & GWI & $\begin{array}{c}\text { Fold Increase } \\
\text { (GWI/Control) }\end{array}$ & $\begin{array}{l}\text { P } \\
\text { Value }\end{array}$ & Control & GWI & $\begin{array}{c}\text { Fold Increase } \\
\text { (GWI/Control) }\end{array}$ & $\begin{array}{l}\text { P } \\
\text { Value }\end{array}$ \\
\hline Hippocampus & 8.0 & 24.8 & 3.1 & 0.001 & 3.2 & 12.2 & 3.8 & 0.0004 \\
\hline Cortex & 15.5 & 18.3 & 1.2 & 0.48 & 5.1 & 7.0 & 1.4 & 0.07 \\
\hline Cerebellum & 26.9 & 45.1 & 1.7 & 0.06 & 2.8 & 4.7 & 1.7 & 0.41 \\
\hline
\end{tabular}


Table 2. Biological pathways affected by changes in hippocampal miRNA expression one year after Gulf War-related exposures.

\begin{tabular}{|c|c|c|c|c|c|c|}
\hline Category & Pathway Term & $\begin{array}{l}\text { No. of } \\
\text { Genes }\end{array}$ & Target Genes in Pathway & $\begin{array}{l}\text { Fold } \\
\text { Enrichment }\end{array}$ & P Value & $\begin{array}{l}\text { Benjamin } \\
\text { P Value }\end{array}$ \\
\hline \multicolumn{7}{|c|}{ Rat miR-124 and miR-29b validated and predicted targets } \\
\hline KEGG_PATHWAY & $\begin{array}{l}\text { ECM-receptor } \\
\text { interaction }\end{array}$ & 10 & $\begin{array}{l}\text { COL4A1, ITGA7, COL3A1, COL6A2, LAMC1, AGRN, COL1A1, } \\
\text { COL5A3, COL11A1, COL5A2 }\end{array}$ & 7.58 & 4.90E-06 & $5.38 \mathrm{E}-04$ \\
\hline PANTHER_PATHWAY & $\begin{array}{l}\text { Cadherin } \\
\text { signaling } \\
\text { pathway }\end{array}$ & 13 & $\begin{array}{l}\text { PCDHA6, PCDHA7, PCDHA8, PCDHA9, PCDHA2, PCDHA3, } \\
\text { PCDH21, PCDHA4, PCDHA5, CDH2, PCDHA1, PCDHAC2, } \\
\text { PCDHAC1, CDH9, PCDHA10, PCDHA11, PCDHA12, PCDHA13 }\end{array}$ & 3.65 & $1.28 \mathrm{E}-04$ & 0.008 \\
\hline PANTHER_PATHWAY & $\begin{array}{l}\text { Integrin } \\
\text { signaling } \\
\text { pathway }\end{array}$ & 15 & $\begin{array}{l}\text { COL4A1, COL3A1, COL5A3, COL5A2, COL4A5, COL9A1, ARPC1B, } \\
\text { MAPK14, ITGA7, LCK, COL6A2, SHC1, COL1A1, LAMC1, COL11A1 }\end{array}$ & 3.13 & $1.53 \mathrm{E}-04$ & 0.005 \\
\hline KEGG_PATHWAY & Focal adhesion & 12 & $\begin{array}{l}\text { COL4A1, PGF, ITGA7, COL3A1, COL6A2, MYLK2, SHC1, LAMC1, } \\
\text { COL1A1, COL5A3, COL11A1, COL5A2 }\end{array}$ & 3.78 & $2.62 \mathrm{E}-04$ & 0.014 \\
\hline PANTHER_PATHWAY & $\begin{array}{l}\text { Wnt signaling } \\
\text { pathway }\end{array}$ & 17 & $\begin{array}{l}\text { PCDHA6, PCDHA7, PCDHA8, PCDHA9, PCDHA2, PCDHA3, } \\
\text { PCDH21, PCDHA4, PCDHA5, PCDHA1, CDH2, ITPR3, PCDHAC2, } \\
\text { PCDHAC1, DACT1, CDH9, PCDHA10, PCDHA11, CHP, PCDHA12, } \\
\text { PCDHA13, AXIN1 }\end{array}$ & 2.27 & 0.002 & 0.036 \\
\hline \multicolumn{7}{|c|}{ Human miR-124 and miR-29b validated and predicted targets } \\
\hline KEGG_PATHWAY & Focal adhesion & 18 & $\begin{array}{l}\text { CAV2, CAV1, TLN1, COL4A1, PGF, ROCK2, COL3A1, COL2A1, } \\
\text { COL5A3, ITGB1, COL5A2, CDC42, CCND2, PDGFC, LAMC1, } \\
\text { COL1A1, COL11A1, PIK3R1 }\end{array}$ & 3.93 & 2.03E-06 & $2.62 \mathrm{E}-04$ \\
\hline PANTHER_PATHWAY & $\begin{array}{l}\text { Integrin } \\
\text { signaling } \\
\text { pathway }\end{array}$ & 19 & $\begin{array}{l}\text { CAV1, COL4A1, COL3A1, COL2A1, COL5A3, COL5A2, ITGB1, } \\
\text { PTPN12, COL4A5, CDC42, COL9A1, ARPC1B, COL19A1, MAPK14, } \\
\text { ARAF, LAMC1, COL1A1, COL11A1, PIK3R1 }\end{array}$ & 3.22 & $9.48 \mathrm{E}-06$ & 7.39E-04 \\
\hline REACTOME_PATHWAY & $\begin{array}{l}\text { Signaling by } \\
\text { PDGF }\end{array}$ & 9 & $\begin{array}{l}\text { COL9A1, COL4A1, COL3A1, PDGFC, COL2A1, COL1A1, COL5A2, } \\
\text { PIK3R1, COL4A5 }\end{array}$ & 6.21 & 6.97E-05 & 0.003 \\
\hline REACTOME_PATHWAY & Axon guidance & 8 & $\begin{array}{l}\text { COL9A1, COL4A1, COL3A1, COL2A1, COL1A1, COL5A2, SPTAN1, } \\
\text { COL4A5 }\end{array}$ & 7.21 & $8.57 \mathrm{E}-05$ & 0.002 \\
\hline REACTOME_PATHWAY & $\begin{array}{l}\text { Integrin cell } \\
\text { surface } \\
\text { interactions }\end{array}$ & 9 & $\begin{array}{l}\text { F11R, TLN1, COL4A1, FBN1, COL2A1, LAMC1, COL1A1, ITGB1, } \\
\text { COL4A5 }\end{array}$ & 4.90 & $3.73 \mathrm{E}-04$ & 0.005 \\
\hline KEGG_PATHWAY & $\begin{array}{l}\text { ECM-receptor } \\
\text { interaction }\end{array}$ & 9 & $\begin{array}{l}\text { COL4A1, COL3A1, COL2A1, LAMC1, COL1A1, COL5A3, COL5A2, } \\
\text { COL11A1, ITGB1 }\end{array}$ & 4.70 & $5.58 \mathrm{E}-04$ & 0.035 \\
\hline
\end{tabular}


Table 3. The twenty most abundant miRNAs among the plasma exosome RNAs from pooled GWI and control samples.

\begin{tabular}{lll}
\hline miRNA & $\begin{array}{l}\text { Raw Read Counts } \\
\text { Controls }\end{array}$ & $\begin{array}{l}\text { Raw Read Counts } \\
\text { GWI }\end{array}$ \\
\hline rno-miR-16-5p & 11515 & 2776 \\
rno-miR-122-5p & 5727 & 12076 \\
rno-miR-143-3p & 4776 & 1734 \\
rno-miR-22-3p & 3930 & 1939 \\
rno-miR-21-5p & 2759 & 850 \\
rno-miR-24-3p & 2488 & 1373 \\
rno-miR-19b-3p & 1863 & 646 \\
rno-miR-30a-5p & 1757 & 702 \\
rno-miR-30d-5p & 1607 & 1329 \\
rno-miR-192-5p & 1567 & 1078 \\
rno-mir-92a-3p & 1538 & 1743 \\
rno-miR-320-3p & 1517 & 2555 \\
rno-mir-133a-3p & 1353 & 667 \\
rno-miR-26a-5p & 1347 & 521 \\
rno-let-7c-5p & 1254 & 1307 \\
rno-miR-29a-3p & 1191 & 511 \\
rno-mir-126a & 1154 & 450 \\
rno-let-7b-5p & 1118 & 1566 \\
rno-let-7g-5p & 1066 & 318 \\
rno-miR-27b-3p & 1006 & 359 \\
\hline
\end{tabular}


Table 4. Inflammatory cytokine protein levels in brain lysates one year after completion of the 28-day exposure regimen.

\begin{tabular}{|c|c|c|c|c|c|c|c|c|c|}
\hline Cytokine & \multicolumn{3}{|c|}{ Hippocampus } & \multicolumn{3}{|c|}{ Cortex } & \multicolumn{3}{|c|}{ Cerebellum } \\
\hline & GWI & Control & $\begin{array}{l}* \\
\text { Value } \\
\end{array}$ & $\underline{\text { GWI }}$ & Control & $\begin{array}{l}\text { P* } \\
\text { Value }\end{array}$ & $\underline{\text { GWI }}$ & Control & $\begin{array}{l}\mathbf{P}^{*} \\
\text { Value }\end{array}$ \\
\hline TNF- $\alpha$ & $72.1(5.3)$ & $63.8(3.3)$ & 0.19 & $114.8(7.0)$ & $75.5(1.8)$ & $<0.001$ & $79.8(6.7)$ & $120.8(10.4)$ & 0.007 \\
\hline IL-1 $\beta$ & ND & ND & --- & ND & ND & --- & $42.1(3.5)$ & $39.6(5.2)$ & 0.71 \\
\hline IL-6 & $6496(787)$ & $7874(560)$ & 0.17 & $8270(341)$ & $7092(485)$ & 0.08 & $11771(718)$ & $11475(1100)$ & 0.83 \\
\hline
\end{tabular}

Values are expressed as mean(SEM) pg cytokine/mg protein. ND, below detectable limits. *t test. 
Table 5. Inflammatory cytokine mRNA expression in the brain one year after completion of the 28-day exposure regimen.

\begin{tabular}{|c|c|c|c|c|c|c|}
\hline \multirow[t]{2}{*}{ Cytokine } & \multicolumn{2}{|c|}{ Hippocampus } & \multicolumn{2}{|l|}{ Cortex } & \multicolumn{2}{|c|}{ Cerebellum } \\
\hline & $\begin{array}{l}\text { GWI vs. Control } \\
\text { Fold Regulation }\end{array}$ & $\begin{array}{l}\text { P } \\
\text { Value }\end{array}$ & $\begin{array}{l}\text { GWI vs. Control } \\
\text { Fold Regulation }\end{array}$ & $\begin{array}{l}\text { P } \\
\text { Value }\end{array}$ & $\begin{array}{l}\text { GWI vs. Control } \\
\text { Fold Regulation }\end{array}$ & $\begin{array}{l}\text { P } \\
\text { Value }\end{array}$ \\
\hline TNF- $\alpha$ & 1.65 & 0.02 & 1.86 & 0.16 & -1.12 & 0.93 \\
\hline IL-1 $\beta$ & -1.16 & 0.71 & -1.11 & 0.87 & 2.02 & 0.005 \\
\hline IL-6 & 1.14 & 0.78 & -1.04 & 0.45 & 1.19 & 0.91 \\
\hline
\end{tabular}


Table 6. ED1-positive cells (a marker of activated microglia) one year after completion of the 28-day exposure regimen.

\begin{tabular}{|c|c|c|c|c|c|c|c|c|}
\hline \multicolumn{3}{|c|}{ Hippocampus } & \multicolumn{3}{|c|}{ Cortex } & \multicolumn{3}{|c|}{ Cerebellum } \\
\hline GWI & Control & $\begin{array}{l}P^{*} \\
\text { Value }\end{array}$ & $\underline{\text { GWI }}$ & Control & $\begin{array}{l}\mathrm{P}^{*} \\
\text { Value }\end{array}$ & $\underline{\text { GWI }}$ & Control & $\begin{array}{l}\mathrm{P}^{*} \\
\text { Value }\end{array}$ \\
\hline $5.23(0.41)$ & $6.77(0.70)$ & 0.07 & $2.61(0.29)$ & $2.60(0.41)$ & 0.99 & $3.64(0.41)$ & $3.37(0.30)$ & 0.65 \\
\hline
\end{tabular}

Values are expressed as mean(SEM) ED1-positive cells per $400 \times$ field. *t test. 